\title{
Conformal Nets V: Dualizability
}

\author{
Arthur Bartels $^{1}$, Christopher L. Douglas ${ }^{2}$ (D) André Henriques $^{2}$ \\ 1 Mathematisches Institut, Westfälische Wilhelms-Universität Münster, Einsteinstr. 62, 48149 Münster, \\ Deutschland. E-mail: bartelsa@wwu.de \\ http://www.math.uni-muenster.de/u/bartelsa \\ 2 Mathematical Institute, University of Oxford, Woodstock Road, Oxford OX2 6GG, UK. \\ E-mail: cdouglas@maths.ox.ac.uk; andre.henriques@maths.ox.ac.uk \\ http://people.maths.ox.ac.uk/cdouglas; http://www.andreghenriques.com
}

Received: 8 March 2021 / Accepted: 24 August 2021

Published online: 21 February 2022 - (C) The Author(s) 2022

\begin{abstract}
We prove that finite-index conformal nets are fully dualizable objects in the 3-category of conformal nets. Therefore, assuming the cobordism hypothesis applies, there exists a local framed topological field theory whose value on the point is any finite-index conformal net. Along the way, we prove a Peter-Weyl theorem for defects between conformal nets, namely that the annular sector of a finite defect is the sum of every sector tensored with its dual.
\end{abstract}

\section{Contents}

1. Defect Algebras Acting on Annuli and Discs . . . . . . . . . . . . . . 6

1.A The Hilbert space for a bicolored annulus . . . . . . . . . . . . . 6

1.B A Peter-Weyl theorem for defects . . . . . . . . . . . . . . . . . 9

1.C Extending defects to bicolored 1-manifolds . . . . . . . . . . . 13

2. A Characterization of Dualizable Conformal Nets . . . . . . . . . . . . 15

2.A Involutions on nets, defects, sectors, and intertwiners . . . . . . . . 15

2.B The snake interchange isomorphism for defects . . . . . . . . . . 16

2.C Finite nets are dualizable . . . . . . . . . . . . . . . . . . . 19

2.D Dualizable nets are finite ................... 23

Appendix A: Disintegrating Sectors Between Finite Defects . . . . . . . . . . . 28

Appendix B: A Variant Vertical Composition . . . . . . . . . . . . 30

References ...................... 30

\section{Introduction}

A finite-dimensional Hilbert space $H$ is dualizable in the sense that there is a Hilbert space $H^{*}$ together with evaluation and coevaluation morphisms ev $: H \otimes H^{*} \rightarrow \mathbb{C}$ and coev $: \mathbb{C} \rightarrow H^{*} \otimes H$ such that the identity id $_{H}$ can be recovered as the composite 
$\left(\operatorname{coev} \otimes \mathrm{id}_{H}\right) \circ\left(\mathrm{id}_{H} \otimes \mathrm{ev}\right)$, and the identity $\mathrm{id}_{H^{*}}$ can be recovered as a similar composite; indeed, every dualizable Hilbert space is finite-dimensional.

The 2-category vN of von Neumann algebras deloops the category Hilb of Hilbert spaces in the sense that $\operatorname{Hom}_{\mathrm{VN}}(1,1) \cong \mathrm{Hilb}$. If a von Neumann algebra $A$ is a finite direct sum of type $I$ factors, then it is fully dualizable in the sense that there is a von Neumann algebra $A^{o p}$ together with evaluation bimodule $A \otimes A^{o p} H_{\mathbb{C}}$ and coevaluation bimodule $\mathbb{C} H_{A^{o p} \otimes A}$ such that the identity bimodule ${ }_{A} L^{2}(A)_{A}$ can be recovered as a composite of the evaluation and coevaluation (and the identity bimodule for $A^{o p}$ can be similarly recovered), and such that the evaluation and coevaluation bimodules themselves admit adjoints. A fully dualizable von Neumann algebra is in fact necessarily a finite direct sum of type $I$ factors. More generally, full dualizability functions as a strong finiteness condition on the objects of a higher category.

The 3-category $\mathrm{CN}$ of conformal nets deloops the 2-category vN of von Neumann algebras, in the sense that $\operatorname{Hom}_{\mathrm{CN}}(1,1) \cong \mathrm{vN}$ [BDH19, Proposition 1.22]. In this paper, the fifth in a series [BDH15,BDH17,BDH19,BDH18] concerning the 3-category of conformal nets, we investigate the dualizability properties of conformal nets and their defects and sectors. Our main result is that a conformal net is fully dualizable if (Theorem $\mathrm{B}$ below) and only if (Theorem $\mathrm{C}$ below) it has finite index.

Dualizability. Recall that two $i$-morphisms $F: B \rightarrow A$ and $G: A \rightarrow B$ in an $n$-category $(i<n)$ are called adjoint (or dual), denoted $F \dashv G$, if there exist $(i+1)$ morphisms, the unit $s: \mathrm{id}_{B} \rightarrow G \circ F$ and the counit $r: F \circ G \rightarrow \mathrm{id}_{A}$ such that the composite $\left(\operatorname{id}_{G} \odot r\right) \circ\left(s \odot \mathrm{id}_{G}\right)$ is equivalent to $\mathrm{id}_{G}$ and the composite $\left(r \odot \mathrm{id}_{F}\right) \circ\left(\mathrm{id}_{F} \odot s\right)$ is equivalent to $\mathrm{id}_{F}$; we say that $F$ admits $G$ as its right adjoint, or equivalently that $G$ admits $F$ as its left adjoint. (Here $\odot$ denotes functorial composition of $(i+1)$-morphisms in the direction of $i$-morphisms.) Similarly, two objects $f$ and $g$ in a symmetric monoidal $n$-category are called dual if there exist 1-morphisms, the coevaluation $s: 1 \rightarrow f \otimes g$ and the evaluation $r: g \otimes f \rightarrow 1$, such that the composite $\left(r \otimes \mathrm{id}_{g}\right) \circ\left(\mathrm{id}_{g} \otimes s\right)$ is equivalent to $\mathrm{id}_{g}$ and the composite $\left(\mathrm{id}_{f} \otimes r\right) \circ\left(s \otimes \mathrm{id}_{f}\right)$ is equivalent to $\mathrm{id}_{f}$. (Note that by convention, the monoidal structure $\otimes$ has a geometric rather than functorial order of composition.)

An $i$-morphism $F: A \rightarrow B$ in an $n$-category $(i<n)$ is called fully dualizable if there is an infinite chain of adjunctions $\cdots F^{L L} \dashv F^{L} \dashv F \dashv F^{R} \dashv F^{R R} \dashv \cdots$ such that every unit and counit morphism in each of the adjunctions in that chain itself admits a similar infinite chain of adjunctions, such that every unit and counit morphism in each of the adjunctions in all of those chains in turn admits an infinite chain of adjunctions, and so on until one reaches a chain of $(n-1)$-morphisms, at which point the conditions stop. (We refer to an $(n-1)$-morphism that has an infinite chain of left and right adjoints, and is therefore fully dualizable, simply as 'dualizable'.) Similarly, an object in a symmetric monoidal $n$-category is fully dualizable (also called ' $n$-dualizable') if it admits a dual and the coevaluation and evaluation morphisms are fully dualizable. A symmetric monoial $n$-category is said to have all duals if every object is fully dualizable and every $i$ morphism $(i<n)$ is fully dualizable. (Note that the notions of fully dualizable and of having all duals do not depend on the exact model one chooses for symmetric monoidal $n$-categories, because the dualizability conditions can be phrased entirely in terms of homotopy 2-categories canonically associated to the $n$-category. For a more detailed discussion of the notion of dualizability, see [DSPS17, Appendix A].)

The cobordism hypothesis [BD95,Lur09, AF17] ensures that for any fully dualizable object $c$ in a symmetric monoidal $n$-category $\mathcal{C}$, there is a local framed topological field 
theory $F_{c}:$ Bord $_{n}^{\mathrm{fr}} \rightarrow \mathcal{C}$ whose value on the positively framed point is $c .{ }^{1}$ In particular, for any such object, there is an associated framed $n$-manifold invariant.

Finiteness. We will investigate the dualizability of objects and morphisms in the symmetric monoidal 3-category of conformal nets. To that end, we introduce notions of 'finiteness' for nets, defects, and sectors, arranged in such a way that finiteness ensures both the existence of a dual (or adjoint) and in turn the finiteness of the coevaluation and evaluation (or unit and counit) morphisms. We will therefore be able to successively establish that finiteness implies dualizability for sectors, defects, and conformal nets.

Consider the following subintervals of the standard circle:

$$
\begin{array}{ll}
S_{\top}^{1}:=\left\{z \in S^{1} \mid \Im \mathrm{m}(z) \geq 0\right\}, & S_{\dashv}^{1}:=\left\{z \in S^{1} \mid \mathfrak{R e}(z) \geq 0\right\}, \\
S_{\perp}^{1}:=\left\{z \in S^{1} \mid \Im \mathrm{m}(z) \leq 0\right\}, & S_{\vdash}^{1}:=\left\{z \in S^{1} \mid \mathfrak{R e}(z) \leq 0\right\} .
\end{array}
$$

Moreover, let $I_{1}, \ldots, I_{4} \subset S^{1}$ be the subintervals indicated here:

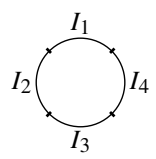

When appropriate, we equip the standard circle $S^{1}$ with its standard bicoloring $S_{\circ}^{1}=S_{\vdash}^{1}$, $S_{\bullet}^{1}=S_{\dashv}^{1}$, and give $I_{1}, \ldots, I_{4}$ the induced bicoloring, so that $I_{1}$ and $I_{3}$ are genuinely bicolored, $I_{2}$ is white, and $I_{4}$ is black.

We work with coordinate-free nets [BDH15, Definition 1.1]; thus a conformal net $\mathcal{A}$ is a functor from the category of intervals to the category of von Neumann algebras. The vacuum sector $H_{0}(\mathcal{A})$ is a Hilbert space equipped with actions of the algebras $\mathcal{A}(I)$, where $I$ varies over the subintervals of the standard circle [BDH15, Definition 1.12]. (Note that the vacuum sector $H_{0}(\mathcal{A})$ is not extra structure on a conformal net, but is defined explicitly in terms of the von Neumann algebras associated to intervals.) The split property of $\mathcal{A}$ gives additional actions associated to disjoint unions of subintervals of the circle. In particular, the algebra $\mathcal{A}\left(I_{1} \cup I_{3}\right) \cong \mathcal{A}\left(I_{1}\right) \otimes \mathcal{A}\left(I_{3}\right)$ acts on $H_{0}(\mathcal{A})$, and similarly for $I_{2} \cup I_{4}$. Given nets $\mathcal{A}$ and $\mathcal{B}$, an $\mathcal{A}$ - $\mathcal{B}$-defect ${ }_{\mathcal{A}} D_{\mathcal{B}}$ is a functor from the category of bicolored intervals to von Neumann algebras [BDH19, Definition 1.7]. The two colors of the bicolored intervals correspond to the source and target nets $\mathcal{A}$ and $\mathcal{B}$ of the defect. The vacuum sector $H_{0}(D)$ is a Hilbert space equipped with actions of the algebras $D(I)$, where $I$ varies over the subintervals of the standard bicolored circle. (As for nets, the vacuum sector $H_{0}(D)$ is not additional structure on a defect, but is constructed from the given von Neumann algebras.)

We henceforth assume that all conformal nets and defects are semisimple, that is finite direct sums of irreducible ones; (a conformal net or defect is irreducible if it does not admit a non-trivial direct sum decomposition).

\footnotetext{
1 See the section on 'Manifold invariants' below, and in particular Footnote 3, for a discussion of the applicability of the cobordism hypothesis to the symmetric monoidal 3-category of conformal nets.
} 


\section{Definition 0.2.}

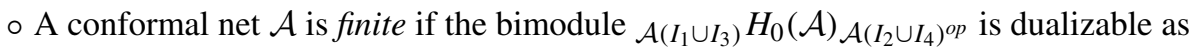
a morphism in the 2-category of von Neumann algebras. ${ }^{2}$

$\circ$ A defect ${ }_{\mathcal{A}} D_{\mathcal{B}}$ between finite conformal nets is finite if the action of the algebraic tensor product $D\left(I_{1}\right) \otimes_{\text {alg }} D\left(I_{3}\right)$ on $H_{0}(D)$ extends to the spatial tensor product $D\left(I_{1}\right) \bar{\otimes} D\left(I_{3}\right)$, that is, if the Hilbert space $H_{0}(D)$ is split as a $D\left(I_{1}\right)-D\left(I_{3}\right)^{o p}$ bimodule.

○ A $D$ - $E$-sector $H$ between defects $D$ and $E$, is finite if the bimodule ${ }_{D\left(S_{\top}^{1}\right)} H_{E\left(S_{\perp}^{1}\right)^{o p}}$ is dualizable as a morphism in the 2-category of von Neumann algebras.

Note that, because there is a contravariant involution on the 2-morphisms of the 2category of von Neumann algebras (namely the adjoint map of Hilbert spaces), a left adjoint bimodule is also a right adjoint bimodule and vice versa; thus for a bimodule to be dualizable it suffices that it admit a single adjoint.

Statement of results. In order to construct adjunctions for defects, we will need to understand the Hilbert space assigned by a defect to a bicolored annulus. To that end, we prove the following Peter-Weyl annular decomposition theorem for defects, generalizing the Kawahigashi-Longo-Müger theorem for conformal nets [KLM01, Theorem 9]. Given

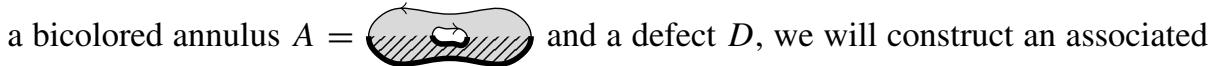
Hilbert space $H_{a n n}(D)$, considered as a ' $\partial A$-sector', that is, a representation $\rho$ of the collection of algebras $\{D(I)\}$ for $I$ a subinterval of the boundary $\partial A$ (a disconnected 1-manifold), subject to the following isotony and locality axioms:

$$
\begin{aligned}
& \text { (isotony): }\left.\quad I \subset J \Rightarrow \rho_{D(J)}\right|_{D(I)}=\rho_{D(I)} \text {, } \\
& \text { (locality): } \stackrel{\circ}{\mathrm{I}} \cap \stackrel{\circ}{J}=\emptyset \Rightarrow\left[\rho_{D(I)}, \rho_{D(J)}\right]=0 \text {. }
\end{aligned}
$$

Let $\Delta_{D}$ be the set of isomorphism classes of irreducible $D$ - $D$-sectors, with the sector associated to $\lambda \in \Delta_{D}$ denoted $H_{\lambda}$. Let $\bar{\lambda}$ denote the dual isomorphism class, and let $H_{\lambda} \otimes H_{\bar{\lambda}}$ denote the $\partial A$-sector where one circle acts on $H_{\lambda}$ and the other circle acts on $H_{\bar{\lambda}}$.

Theorem A (Peter-Weyl for defects). For a finite irreducible defect $D$, the annular sector $H_{a n n}(D)$ is non-canonically isomorphic to the sum $\bigoplus_{\lambda \in \Delta_{D}} H_{\lambda} \otimes H_{\bar{\lambda}}$ of every sector tensor its dual.

This is proven as Theorem 1.13 in the text. We may depict this result as
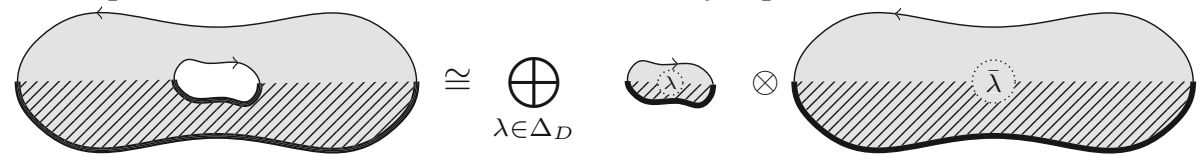

\footnotetext{
2 If $\mathcal{A}$ is irreducible, then this condition is equivalent to the conformal net having finite index, as follows. Recall from [BDH15, Definition 3.1] that the index of a conformal net $\mathcal{A}$ is defined as the minimal index of the inclusion $\mathcal{A}\left(I_{1} \cup I_{3}\right) \subset \mathcal{A}\left(I_{2} \cup I_{4}\right)^{\prime}$. By [BDH14, Proposition 7.5], if this minimal index is finite, then the bimodule $\mathcal{A}\left(I_{1} \cup I_{3}\right) H_{0}(\mathcal{A}) \mathcal{A}\left(I_{2} \cup I_{4}\right)^{o p}$ is dualizable. Conversely, if that bimodule is dualizable, then, by [BDH14, Definition 5.1], its statistical dimension is finite and thus, by [BDH14, Definition 5.10 \& Proposition 7.3], the corresponding minimal index is finite.
} 
Equipped with this and other results about defect annular sectors, we proceed to our main topic of dualizability properties of conformal nets. We show that finite sectors are dualizable; that finite defects are dualizable with finite unit and counit sectors (and hence are fully dualizable); and that finite conformal nets are dualizable with finite evaluation and coevaluation defects (and hence are fully dualizable).

Theorem B (Dualizability of finite nets, defects, and sectors). The 3-category of finite semisimple conformal nets, finite semisimple defects, finite sectors, and intertwiners has all duals.

This result is summarized as Theorem 2.20 in the text, collecting the results of Proposition 2.11, Corollary 2.12, Proposition 2.14, Corollary 2.16, Theorem 2.17, and Corollary 2.19. Theorem A is used (via Proposition 1.21 and Corollary 1.16) in the proof of the crucial Proposition 2.14, in order to construct the unit and counit sectors needed for the adjunction of defects.

Having established that finiteness implies full dualizability, we conversely establish that full dualizability ensures finiteness.

Theorem C (Finiteness of dualizable nets, defects, and sectors). A fully dualizable conformal net, defect, or sector is necessarily finite.

See Corollary 2.12, Proposition 2.22, Theorem 2.25, and Remark 2.31 in the text for the precise statements and proofs. Note that we do not have a 3-category of all notnecessarily-finite conformal nets (because we do not know that the composition of two defects between non-finite nets is again a defect); however the notion of dualizability is still well defined for an arbitrary not-necessarily-finite net (namely as the condition that the canonical evaluation and coevaluation defects both have ambidextrous adjoints with dualizable unit and counit sectors), and therefore it makes sense to claim and prove as we do that a dualizable net is finite.

Manifold invariants. By Theorem B and under the (overwhelmingly plausible but not yet proven) assumption that the cobordism hypothesis applies to the symmetric monoidal 3category of conformal nets constructed in [BDH18], ${ }^{3}$ associated to any finite conformal net there is a 3-dimensional local framed topological field theory whose value on a point is the conformal net. Naturally, one wonders what manifold invariants are given by this topological field theory.

For 1-dimensional manifolds, the conformal net field theory invariants are given, projectively (that is, up to tensoring by an invertible von Neumann algebra), by the extension, constructed in [BDH17, Theorem 1.3], of the conformal net to a functor from 1-manifolds to the category of von Neumann algebras. In particular, the invariant of a circle is the direct sum over irreducible representations of the algebra of bounded operators on the underlying representation space (see [BDH17, Theorem 1.20]). One may also express the invariant of a circle as the colimit in the category of von Neumann algebras of the value of the conformal net on all the subintervals of the circle (see [BDH17, Proposition 1.25]).

3 As the cobordism hypothesis applies most immediately to symmetric monoidal $n$-categories modeled as $\Gamma$-objects in complete $n$-fold Segal spaces [Lur09,CS15], this assumption can be made precise in the form of the following conjecture: there exists a $\Gamma$-object in complete 3-fold Segal spaces $C N^{\prime}$ together with an equivalence of tricategories $E:[\mathrm{CN}] \rightarrow\left[\mathrm{CN}^{\prime}\right]$; here $\mathrm{CN}$ denotes the symmetric monoidal 3-category of finite conformal nets constructed as an internal dicategory in symmetric monoidal categories [BDH18], and the brackets [-] denote the tricategory associated to either the internal dicategory in symmetric monoidal categories or the $\Gamma$-object in complete 3 -fold Segal spaces. 
For 2-dimensional manifolds, the conformal net field theory invariants are given, projectively (that is, up to tensoring by an invertible Hilbert space), by the functor constructed in [BDH17, Theorem 2.18], from 2-manifolds to Hilbert spaces. In particular, the invariant of a closed 2-manifold is given, projectively, by the space of conformal blocks associated to that surface. When the conformal net is $\mathcal{N}_{G, k}$, the one associated to the level $k$ central extension of the loop group $L G$, we expect the invariant is the space of conformal blocks for the corresponding chiral WZW model.

For any finite-index conformal net $\mathcal{A}$, under the aforementioned assumption that the cobordism hypothesis applies, our results provide a complex-valued invariant $Z_{\mathcal{A}}(M)$ of any closed framed 3-manifold $M$. For the loop group conformal net $\mathcal{N}_{G, k}$, the category $\operatorname{Rep}\left(\mathcal{N}_{G, k}\right)$ of representations of the net is thought to be isomorphic to the category $\operatorname{Rep}(L G, k)$ of representations of the loop group $L G$ at level $k$; see [Hen17] for a discussion of this comparison problem and [Gui18, Section 5.1] for progress towards a solution. Provided the representation categories of the conformal net and of the loop group are indeed isomorphic as modular tensor categories, then we expect the 3-manifold invariant $Z_{\mathcal{N}_{G, k}}(M)$ determined by the conformal-net-valued local field theory is the Reshetikhin-Turaev invariant of $M$ associated to the modular tensor category $\operatorname{Rep}(L G, k)$ of representations of the associated loop group.

\section{Defect Algebras Acting on Annuli and Discs}

We will, later in Sect. 2, interpret the fusion of a defect and its adjoint as associating an algebra to an interval with not just a single transition point from white to black, but instead two: one from white to black, and then one back to white. To construct the unit and counit of the adjunction, we will need an action of this larger algebra on the vacuum sector of the original defect. We will construct such an action by first constructing an action on a Hilbert space associated to an annulus and then "plugging the hole" of the annulus with a vacuum sector.

Working up to those constructions, in this section we study the Hilbert space associated to a bicolored annulus; we prove a Peter-Weyl theorem decomposing the defect annular Hilbert space as a sum of tensor products of sectors and their duals, and we define the algebras associated to arbitrary bicolored 1-manifolds.

1.A. The Hilbert space for a bicolored annulus. Given a finite defect $D$ between finite conformal nets, the bimodule

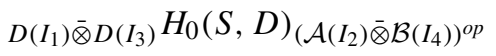

is always dualizable (see [BDH19, Proposition 3.18] and Footnote 2). Here $S$ is a bicolored circle decomposed into intervals $I_{1}, \ldots, I_{4}$, as in $(0.1)$, and the vacuum sector $H_{0}(S, D)$ is described in [BDH19, Notation 1.14]. Let $-S,-I_{1}, \ldots,-I_{4}$ be the same manifolds with the reverse orientations. The following result explicitly identifies the dual, generalizing the corresponding result for conformal nets [BDH15, Lemma 3.4]:

Lemma 1.2. Under the canonical identifications $\left(D\left(-I_{1}\right) \bar{\otimes} D\left(-I_{3}\right)\right)^{o p} \cong D\left(I_{1}\right) \bar{\otimes}$ $D\left(I_{3}\right)$ and $\left(\mathcal{A}\left(-I_{2}\right) \bar{\otimes} \mathcal{B}\left(-I_{4}\right)\right)^{o p} \cong \mathcal{A}\left(I_{2}\right) \bar{\otimes} \mathcal{B}\left(I_{4}\right)$, the dual of the bimodule (1.1) is given by

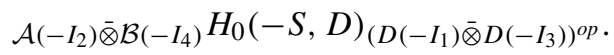


Proof. We assume without loss of generality that $S$ is the standard bicolored circle. Let us write $S^{1}=K_{1} \cup \cdots \cup K_{6}$, with $K_{1}=I_{4} \cap S_{\text {个 }}^{1}, K_{2}=I_{1}, K_{3}=I_{2} \cap S_{\text {个 }}^{1}, K_{4}=I_{2} \cap S_{\perp}^{1}$, $K_{5}=I_{3}, K_{6}=I_{4} \cap S_{\perp}^{1}$

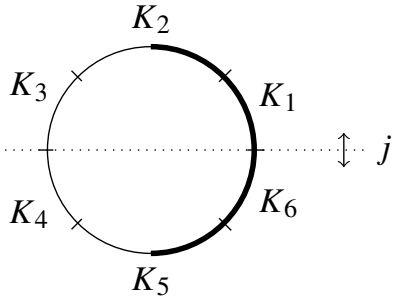

and let $j$ be the reflection that exchanges $S_{\top}^{1}$ and $S_{\perp}^{1}$. For any interval $I$, we abbreviate $D(j): D(I) \rightarrow D(j(I))^{o p}$ by $j_{*}$ and let $A:=D\left(S_{\top}^{1}\right)$. By definition, $H_{0}(S, D)=$ $L^{2}(A)$ with actions

$\left(a_{1} \otimes a_{2} \otimes a_{3} \otimes a_{4} \otimes a_{5} \otimes a_{6}\right) \cdot \xi:=\left(a_{1} a_{2} a_{3}\right) \xi j_{*}\left(a_{4} a_{5} a_{6}\right)^{o p}, \quad a_{i} \in D\left(K_{i}\right)$.

Here $a^{o p} \in A^{o p}$ is the element $a \in A$ viewed as an element of $A^{o p}$. By [BDH14, Corollary 6.12], the dual of $H_{0}$ is its complex conjugate $\overline{H_{0}(S, D)}=\overline{L^{2}(A)}$, with actions $b \cdot \bar{\xi} \cdot a=\overline{a^{*} \cdot \xi \cdot b^{*}}$ for $a \in D\left(I_{1}\right) \bar{\otimes} D\left(I_{3}\right)$ and $b \in\left(\mathcal{A}\left(I_{2}\right) \bar{\otimes} \mathcal{B}\left(I_{4}\right)\right)^{o p}$. We rewrite it as

$$
\begin{aligned}
\left(a_{1} \otimes a_{3} \otimes a_{4} \otimes a_{6}\right) \cdot \bar{\xi} \cdot\left(a_{2}^{o p}\right. & \left.\otimes a_{5}^{o p}\right)=\overline{\left(a_{2}^{o p *} \otimes a_{5}^{o p *}\right) \cdot \xi \cdot\left(a_{1}^{*} \otimes a_{3}^{*} \otimes a_{4}^{*} \otimes a_{6}^{*}\right)} \\
& =\overline{\left(a_{1}^{o p *} \otimes a_{2}^{o p *} \otimes a_{3}^{o p *} \otimes a_{4}^{o p *} \otimes a_{5}^{o p *} \otimes a_{6}^{o p *}\right) \cdot \xi} \\
& =\overline{\left(a_{1}^{o p *} a_{2}^{o p *} a_{3}^{o p *}\right) \xi j_{*}\left(a_{4}^{*} a_{5}^{*} a_{6}^{*}\right)}
\end{aligned}
$$

for $a_{i} \in D\left(K_{i}\right)$.

On the other hand, $H_{0}(-S, D):=L^{2}\left(D\left(-S_{\top}^{1}\right)\right) \cong L^{2}\left(A^{o p}\right)$ has actions $\left(b_{1} \otimes b_{2} \otimes\right.$ $\left.b_{3} \otimes b_{4} \otimes b_{5} \otimes b_{6}\right) \cdot \eta:=\left(b_{1} b_{2} b_{3}\right) \eta j_{*}\left(b_{4} b_{5} b_{6}\right)^{o p}$ for $b_{i} \in D\left(K_{i}\right)^{o p}$ and $\eta \in L^{2}\left(A^{o p}\right)$. Using the canonical identification $\eta \mapsto \eta^{o p}$ between $L^{2}\left(A^{o p}\right)$ and $L^{2}(A)$ that exchanges the left $A^{o p}$-module structure with the right $A$-module structure and the right $A^{o p}$-module structure with the left $A$-module structure, this becomes

$$
\left[\left(a_{1} \otimes a_{2} \otimes a_{3} \otimes a_{4} \otimes a_{5} \otimes a_{6}\right) \cdot \xi\right]^{o p}=j_{*}\left(a_{4} a_{5} a_{6}\right) \xi^{o p}\left(a_{1}^{o p} a_{2}^{o p} a_{3}^{o p}\right)
$$

for $a_{i} \in D\left(K_{i}\right)$ and $\xi \in L^{2}(A)$. Finally, the isomorphism intertwining (1.4) and (1.5) is given by the modular conjugation $J: \overline{L^{2}(A)} \rightarrow L^{2}(A)$.

We now investigate what happens when we glue two vacuum sectors along a pair of intervals. Instead of viewing the vacuum sector $H_{0}(S, D)$ as being associated to a bicolored circle $S$ as in [BDH19, Notation 1.14], we shall think of it as being associated to a bicolored disk:

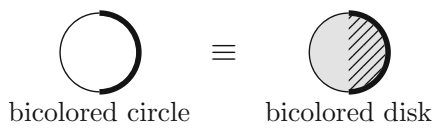

This is merely a change of notation, not of content. (Note that, as in [BDH19], the Hilbert space $H_{0}(S, D)$ is only well defined up to non-canonical isomorphism.) 
Given two genuinely bicolored disks $\mathbb{D}_{l}, \mathbb{D}_{r}$, we investigate two ways of gluing them together into annulus. Decompose each of their boundaries into four intervals $S_{l}:=\partial \mathbb{D}_{l}=I_{1} \cup \cdots \cup I_{4}$ and $S_{r}:=\partial \mathbb{D}_{r}=I_{5} \cup \cdots \cup I_{8}$, where $I_{1}, I_{3}, I_{5}, I_{7}$ are genuinely bicolored, $I_{4}, I_{6}$ are white, and $I_{2}, I_{8}$ are black. If we glue $\mathbb{D}_{l}$ to $\mathbb{D}_{r}$ along diffeomorphisms $I_{1} \leftrightarrow I_{5}$ and $I_{3} \leftrightarrow I_{7}$, we get the following bicolored annulus:

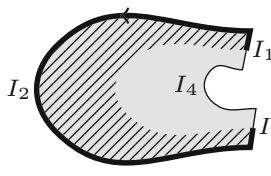

$\mathbb{D}_{l}$
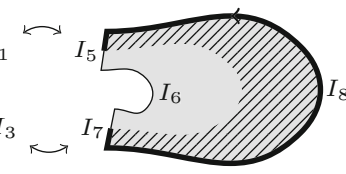

$\mathbb{D}_{r}$

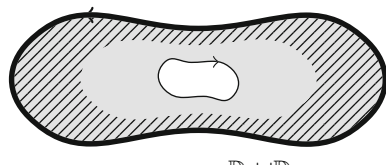

$\mathbb{D}_{l} \cup \mathbb{D}_{r}$

If $D$ is a finite defect, then the action of $D\left(I_{1}\right) \otimes_{\text {alg }} D\left(I_{3}\right)$ on $H_{0}\left(S_{l}, D\right)$ extends to the spatial tensor product $D\left(I_{1}\right) \otimes D\left(I_{3}\right)$. Similarly, the action of $D\left(I_{5}\right) \otimes_{\text {alg }} D\left(I_{7}\right)$ on $H_{0}\left(S_{r}, D\right)$ extends to $D\left(I_{5}\right) \bar{\otimes} D\left(I_{7}\right)$. Identifying $D\left(I_{5}\right) \bar{\otimes} D\left(I_{7}\right)$ with $D\left(I_{1}\right) \bar{\otimes} D\left(I_{3}\right)^{o p}$ via the diffeomorphism, we can then associate a Hilbert space to the annulus (1.6) as follows:

$$
H_{0}\left(S_{l}, D\right) \underset{D\left(I_{5}\right) \bar{\otimes} D\left(I_{7}\right)}{\bigotimes} H_{0}\left(S_{r}, D\right)=, \quad H_{0}\left(S_{l}, D\right) \underset{D\left(I_{5}\right)}{\bigotimes} H_{0}\left(S_{r}, D\right) \underset{D\left(I_{3}\right)}{\bigotimes} ;
$$

Here we used cyclic fusion, see [BDH19, Appendix B.III].

Consider now the slightly different situation where $I_{2}, I_{4}, I_{6}, I_{8}$ are genuinely bicolored, $I_{1}, I_{5}$ are white, and $I_{3}, I_{7}$ are black. Once again, we glue $\mathbb{D}_{l}$ to $\mathbb{D}_{r}$ along two diffeomorphisms $I_{1} \leftrightarrow I_{5}$ and $I_{3} \leftrightarrow I_{7}$

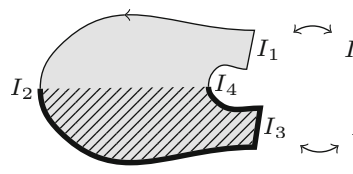

$\mathbb{D}_{l}$

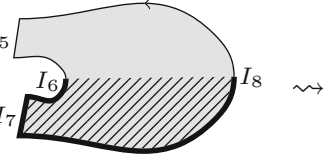

$\mathbb{D}_{\text {r. }}$

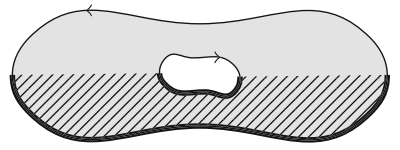

$\mathbb{D}_{l} \cup \mathbb{D}$.

and we associate a Hilbert space to this annulus: ${ }^{4}$

$$
H_{0}\left(S_{l}, D\right) \underset{\mathcal{A}\left(I_{5}\right) \bar{\otimes} \mathcal{B}\left(I_{7}\right)}{\otimes} H_{0}\left(S_{r}, D\right)=, \quad H_{0}\left(S_{l}, D\right) \underset{\mathcal{A}\left(I_{5}\right)}{\otimes} H_{0}\left(S_{r}, D\right) \underset{\mathcal{B}\left(I_{3}\right)}{\otimes} \text {; }
$$

The next lemma describes the structure of the Hilbert spaces associated to the annuli (1.6) and (1.7):

Lemma 1.8. Let ${ }_{\mathcal{A}} D_{\mathcal{B}}$ be a finite irreducible defect, and let $S_{l}, S_{r}, I_{1}, \ldots, I_{8}$ be either as in (1.6) or as in (1.7). Let also $S_{b}:=I_{2} \cup I_{8}$ and $S_{m}:=I_{4} \cup I_{6}$. Then $H_{0}\left(S_{m}, D\right) \otimes$ $H_{0}\left(S_{b}, D\right)$ is a direct summand of

$$
H_{a n n}:=, H_{0}\left(S_{l}, D\right) \underset{D\left(I_{5}\right)}{\otimes} H_{0}\left(S_{r}, D\right) \underset{D\left(I_{3}\right)}{\bigotimes} \text {; ; }
$$

in a way compatible with the actions of $D(J)$ for all $J \subset S_{b}$ and $J \subset S_{m}$. Moreover, $H_{0}\left(S_{m}, D\right) \otimes H_{0}\left(S_{b}, D\right)$ appears with multiplicity 1 inside $H_{\text {ann }}$. (In the case of situation (1.6), by definition $H_{0}\left(S_{m}, D\right)=H_{0}\left(S_{m}, \mathcal{A}\right)$ and $H_{0}\left(S_{b}, D\right)=H_{0}\left(S_{b}, \mathcal{B}\right)$; in this case, we also require that $\mathcal{A}$ and $\mathcal{B}$ be irreducible.)

\footnotetext{
${ }^{4}$ We expect this Hilbert space to be independent of the way we decompose the annulus (for instance, of the number of pieces in the decomposition), but we do not address this independence here.
} 
Proof. Let $A:=D\left(I_{2}\right) \bar{\otimes} D\left(I_{4}\right), B:=\left(D\left(I_{1}\right) \bar{\otimes} D\left(I_{3}\right)\right)^{o p} \cong D\left(I_{5}\right) \bar{\otimes} D\left(I_{7}\right)$, and $C:=\left(D\left(I_{6}\right) \bar{\otimes} D\left(I_{8}\right)\right)^{o p}$, and let us abbreviate

$$
H_{l}:=H_{0}\left(S_{l}, D\right), \quad H_{r}:=H_{0}\left(S_{r}, D\right), \quad H_{b}:=H_{0}\left(S_{b}, D\right), \quad H_{m}:=H_{0}\left(S_{m}, D\right) .
$$

Since $I_{2}, I_{4}, I_{6}, I_{8}$ cover $S_{m} \cup S_{b}$ and $D$ is (and if needed $\mathcal{A}$ and $\mathcal{B}$ are) irreducible, the Hilbert space $H_{m} \otimes H_{b}$ is an irreducible $A$ - $C$-bimodule. We need to show that

$$
\operatorname{hom}_{A, C}\left(H_{m} \otimes H_{b}, H_{a n n}\right)=\operatorname{hom}_{A, C}\left(H_{m} \otimes H_{b}, H_{l} \otimes_{B} H_{r}\right)
$$

is one-dimensional.

The bimodule ${ }_{A} H_{l B}$ is dualizable [BDH19, Propostion 3.18]. Since $D$ is a finite defect, Lemma 1.2 identifies its dual as $\check{H}_{l}:=H_{0}\left(-S_{l}, D\right)$. By the fundamental property of duals (Frobenius reciprocity), we can therefore rewrite (1.9) as

$$
\operatorname{hom}_{B, C}\left(\check{H}_{l} \otimes_{A}\left(H_{m} \otimes H_{b}\right), H_{r}\right) \text {. }
$$

By [BDH17, Lemma A.4] and [BDH19, Lemma 1.15] $\check{H}_{l} \otimes_{A}\left(H_{m} \otimes H_{b}\right)$ is isomorphic to $H_{0}\left(S_{r}, D\right)$. The above expression therefore reduces to $\operatorname{hom}_{B, C}\left(H_{r}, H_{r}\right)$, which is one-dimensional by the irreducibility of the defect $D$.

1.B. A Peter-Weyl theorem for defects. We now prove that there are finitely many isomorphism classes of irreducible $D$ - $D$-sectors (also referred to simply as ' $D$-sectors') for a finite defect $D$, and that every such irreducible sector is finite. This is the analog for sectors between defects of the corresponding fact for representations of conformal nets, and the proof follows the structure of the proof for nets [BDH15, Theorem 3.14].

Let $S$ be a bicolored circle. Recall that an $S$-sector of $D$ is a Hilbert space equipped with actions of the algebras $D(I)$ for all bicolored subintervals $I$ of $S$, subject to the conditions (0.3). As in [BDH15, §1.B], given a $D$-sector $K$ (on the standard bicolored circle) and a bicolored circle $S$, we write $K(S)$ for the $S$-sector $\varphi^{*} K$, where $\varphi: S \rightarrow S^{1}$ is any bicolored diffeomorphism from $S$ to the standard circle. This sector is well defined up to non-canonical isomorphism, by the same argument as in the proof of [BDH15, Proposition 1.14].

Theorem 1.10. Let ${ }_{\mathcal{A}} D_{\mathcal{B}}$ be a finite irreducible defect between finite conformal nets. Then all D-sectors are direct sums of irreducible ones, and all irreducible D-sectors are finite. Moreover, there are only finitely many isomorphism classes of irreducible D-sectors.

Proof. Let $S_{l}, S_{r}, S_{b}, S_{m}$ and $I_{1}, I_{2}, \ldots, I_{8}$ be as follows:

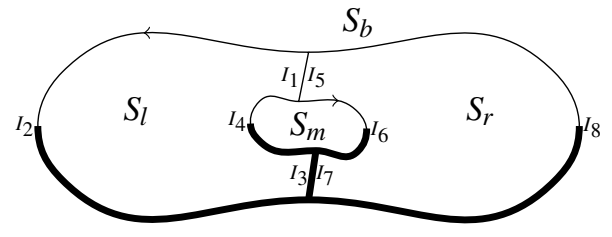

and let $H_{l}=H_{0}\left(S_{l}, D\right), H_{r}=H_{0}\left(S_{r}, D\right), H_{b}=H_{0}\left(S_{b}, D\right), H_{m}=H_{0}\left(S_{m}, D\right)$, and $H_{\text {ann }}:=, H_{l} \bigotimes_{\mathcal{A}\left(I_{5}\right)} H_{r} \otimes_{\mathcal{B}\left(I_{3}\right)} ;$;. Let also 


$$
\begin{aligned}
A & :=D\left(I_{2} \cup I_{4}\right), \quad B:=\left(\mathcal{A}\left(I_{1}\right) \bar{\otimes} \mathcal{B}\left(I_{3}\right)\right)^{o p} \cong \mathcal{A}\left(I_{5}\right) \bar{\otimes} \mathcal{B}\left(I_{7}\right), \quad C:=D\left(I_{6} \cup I_{8}\right)^{o p}, \\
A_{l} & :=D\left(I_{2}\right), \quad A_{m}:=D\left(I_{4}\right)^{o p}, \quad C_{m}:=D\left(I_{6}\right)^{o p}, \quad C_{r}:=D\left(I_{8}\right) .
\end{aligned}
$$

Since ${ }_{A} H_{l B}$ and ${ }_{B} H_{r} C$ are dualizable bimodules, $H_{a n n}=H_{l} \bigotimes_{B} H_{r}$ is dualizable as an $A$ - $C$-bimodule. It therefore splits into finitely many irreducible summands, by Lemma 4.10 and decomposition (5.9) in [BDH14].

Let us now consider $H_{a n n}$ with its actions of $D(I)$ for $I \in \mathrm{INT}_{\circ}^{S_{b}}$. The von Neumann algebra generated by those algebras on $H_{\text {ann }}$ has a finite-dimensional center, since otherwise would contradict the fact that ${ }_{A} H_{\text {ann } C}$ splits into finitely many irreducible summands. We can thus write $H_{a n n}$ as a direct sum of finitely many factorial $S_{b}$-sectors of $D$ :

$$
H_{\text {ann }}=K_{1}\left(S_{b}\right) \oplus \cdots \oplus K_{n}\left(S_{b}\right) .
$$

Here $K_{1}, \ldots, K_{n}$ are $D$-sectors, which we transfer to $S_{b}$ by means of an arbitrary diffeomorphism $S^{1} \cong S_{b}$. (As in the situation without defects [BDH15, Section 3.2], a sector is called factorial if its endomorphism algebra is a factor.)

Given an arbitrary factorial sector $K$, we now show that there exists a $K_{i}$ in the above list to which $K$ is stably isomorphic, i.e., such that $K \otimes \ell^{2} \cong K_{i} \otimes \ell^{2}$. Let us introduce the bicolored circles $S_{2}:=I_{2} \cup_{\partial I_{2}}-I_{2}$ and $S_{4}:=I_{4} \cup_{\partial I_{4}}-I_{4}$. We have isomorphisms $K\left(S_{2}\right) \otimes_{A_{l}} H_{l} \cong K\left(S_{l}\right) \cong H_{l} \bigotimes_{A_{m}} K\left(S_{4}\right)$ of $S_{l}$-sectors (constructed as in [BDH19, Lemma 1.15]). Fusing with $H_{r}$ over $B$, we get an isomorphism

$$
K\left(S_{2}\right) \bigotimes_{A_{l}} H_{a n n} \cong H_{a n n} \bigotimes_{A_{m}} K\left(S_{4}\right) .
$$

Here we used that, by the definition of cyclic fusion, we can also construct $H_{\text {ann }}$ as $H_{l} \otimes_{B} H_{r}$ [BDH19, Appendix B.III]. By Lemma 1.8, it follows that

$$
\begin{aligned}
K\left(S_{b}\right) \otimes H_{m} & \cong\left(K\left(S_{2}\right) \bigotimes_{A_{l}} H_{b}\right) \otimes H_{m} \\
& \cong K\left(S_{2}\right) \bigotimes_{A_{l}}\left(H_{b} \otimes H_{m}\right) \\
& \subset K\left(S_{2}\right) \bigotimes_{A_{l}} H_{\text {ann }} \\
& \cong\left(K\left(S_{2}\right) \bigotimes_{A_{l}} H_{l}\right) \otimes_{D\left(I_{5}\right) \otimes D\left(I_{3}\right)^{o p} H_{r}} \\
& \cong\left(H_{l} \otimes_{A_{l}} K\left(S_{4}\right)\right) \bigotimes_{D\left(I_{5}\right) \otimes D\left(I_{3}\right)^{o p}} H_{r} \cong H_{a n n} \otimes_{A_{m}} K\left(S_{4}\right) .
\end{aligned}
$$

Since $D$ is irreducible, $A_{m}$ is a factor, so $K\left(S_{4}\right)$ and $L^{2} A_{m}$ are stably isomorphic as $A_{m}$-modules, and we get the following (non-canonical) inclusion of $S_{b}$-sectors of $D$ :

$$
\begin{aligned}
K\left(S_{b}\right) \otimes \ell^{2} \cong K\left(S_{b}\right) \otimes H_{m} \otimes \ell^{2} & \subset H_{a n n} \otimes_{A_{m}} K\left(S_{4}\right) \otimes \ell^{2} \\
& \cong H_{a n n} \otimes_{A_{m}} L^{2} A_{m} \otimes \ell^{2} \cong H_{a n n} \otimes \ell^{2},
\end{aligned}
$$

where the first equality is induced by an arbitrary Hibert space isomorphism $\ell^{2} \cong$ $H_{m} \otimes \ell^{2}$. The sector $K\left(S_{b}\right)$ is factorial. It therefore maps to a single summand $K_{i} \otimes \ell^{2}$ of $H_{a n n} \otimes \ell^{2}$. It follows that $K$ and $K_{i}$ are stably isomorphic. In particular, this shows that there are at most finitely many stable isomorphism classes of factorial $D$-sectors on $S_{b}$.

By Lemma A.1, any $D$-sector can be disintegrated into irreducible ones. As a consequence, if there existed a factorial sector of type II or III, then (as in [KLM01, Corollary 58]) there would be uncountably many non-isomorphic irreducible sectors. This is impossible, and so all factorial sectors must be of type $I$. 
We now show all irreducible $D$-sectors are finite. Let us go back to $H_{a n n}$ and analyze it as a $\{D(I)\}_{I \in \mathrm{INT}^{S_{b}}}-\{D(I)\}_{I \in \mathrm{INT}^{S_{m}}}$-representation. Since each summand $K_{i}\left(S_{b}\right)$ in the decomposition (1.12) is a type $I$ factorial $D$-sector, we can write it as $L_{i} \otimes M_{i}$, where $L_{i}$ is an irreducible representation of $\{D(I)\}_{I \in \mathrm{INT}^{\circ}} S_{b}$, and the multiplicity space $M_{i}$ carries a residual action of $\{D(I)\}_{I \in \mathbb{N N T}^{S_{m}}}$. The decomposition (1.12) then becomes

$$
A_{l} \bar{\otimes} A_{m}\left(H_{a n n}\right)_{C_{r} \bar{\otimes} C_{m}} \cong \bigoplus_{i} A_{l} L_{i} C_{r} \otimes A_{m} M_{i} C_{m}
$$

Since $H_{a n n}$ is a dualizable $A$ - $C$-bimodule, the bimodules ${ }_{A_{l}} L_{i} C_{r}$ must also be dualizable. This finishes the argument, as any irreducible $D$-sector on $S_{b}$ is isomorphic to one of the $L_{i}$.

Given a finite irreducible defect $D$, let $\Delta_{D}$ be the finite set of isomorphism classes of irreducible $D$-sectors. For every $\lambda \in \Delta_{D}$, we pick a representative $H_{\lambda}$ of the isomorphism class, which we draw as follows:

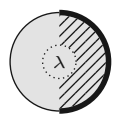

The set $\Delta_{D}$ has an involution $\lambda \mapsto \bar{\lambda}$ given by sending a Hilbert space $H_{\lambda}$ to its complex conjugate $H_{\bar{\lambda}} \cong \bar{H}_{\lambda}$, with actions of $D(I)$ given by

$$
a \bar{\xi}:=\overline{\mathcal{A}(j)\left(a^{*}\right) \xi}
$$

where $j: S^{1} \rightarrow S^{1}$ is the reflection in the horizontal axis (which is color preserving). Note that the isomorphism $H_{\bar{\lambda}} \cong \bar{H}_{\lambda}$ is by no means canonical-see the discussion in [BK01, Remark 2.4.2].

The following Peter-Weyl theorem for defects is analogous to a corresponding annular-sector decomposition theorem for conformal nets by Kawahigashi-LongoMüger [KLM01, Theorem 9], cf also [BDH15, Theorem 3.23]:

Theorem 1.13. Let $D$ be a finite irreducible defect, let $S_{l}, S_{r}, S_{m}, S_{b}$ be as in (1.11), and let

$$
H_{\text {ann }}:=, H_{0}\left(S_{l}, D\right) \underset{\mathcal{A}\left(I_{5}\right)}{\bigotimes} H_{0}\left(S_{r}, D\right) \underset{\mathcal{B}\left(I_{3}\right)}{\bigotimes} \text {; }
$$

We then have a non-canonical isomorphism

$$
H_{a n n} \cong \bigoplus_{\lambda \in \Delta_{D}} H_{\lambda}\left(S_{m}\right) \otimes H_{\bar{\lambda}}\left(S_{b}\right)
$$

of $\left(S_{m} \sqcup S_{b}\right)$-sectors. We draw this isomorphism as
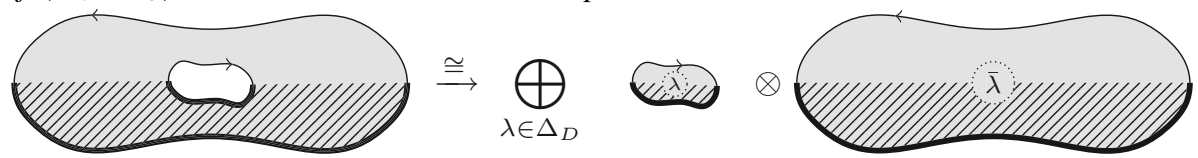
Proof. Let $H_{l}, H_{r}, A, A_{l}, A_{m}, B, C, C_{m}, C_{r}$ be as in the proof of Theorem 1.10, and let $\check{H}_{l}:=H_{0}\left(-S_{l}, D\right)$ be the dual bimodule to ${ }_{A} H_{l B}$ (see Lemma 1.2).

The Hilbert space $H_{a n n}=H_{l} \otimes_{B} H_{r}$ is a finite $A$ - $C$-bimodule and therefore splits into finitely many irreducible summands. By the argument in the proof of Theorem 1.10, each irreducible summand is the tensor product of an irreducible $D$-sector on $S_{m}$ and an irreducible $D$-sector on $S_{b}$. So we can write $H_{a n n}$ as a direct sum

$$
H_{a n n} \cong \bigoplus_{\lambda, \mu \in \Delta_{D}} N_{\lambda \mu} H_{\lambda}\left(S_{m}\right) \otimes H_{\mu}\left(S_{b}\right)
$$

with finite multiplicities $N_{\lambda \mu} \in \mathbb{N}$.

Given $\lambda, \mu \in \Delta_{D}$, we now compute $N_{\lambda \mu}$. Let $K$ be the vertical fusion of $H_{\lambda}$ and $H_{\mu}$. By slight abuse of notation, we abbreviate $H_{\lambda}:=H_{\lambda}\left(S_{m}\right), H_{\mu}:=H_{\mu}\left(S_{b}\right)$, and $K:=K\left(S_{r}\right)$. We then have

$$
\begin{aligned}
\operatorname{hom}_{A, C}\left(H_{\lambda} \otimes H_{\mu}, H_{a n n}\right) & =\operatorname{hom}_{A, C}\left(H_{\lambda} \otimes H_{\mu}, H_{l} \otimes_{B} H_{r}\right) \\
& =\operatorname{hom}_{B, C}\left(\check{H}_{l} \otimes_{A}\left(H_{\lambda} \otimes H_{\mu}\right), H_{r}\right) \\
& =\operatorname{hom}_{B, C}\left(H_{\lambda} \otimes_{A_{m}^{o p}} \check{H}_{l} \otimes_{A_{l}} H_{\mu}, H_{r}\right) \\
& =\operatorname{hom}_{B, C}\left(K, H_{r}\right) \\
& = \begin{cases}\mathbb{C} & \text { if } \mu=\bar{\lambda} \\
0 & \text { otherwise. }\end{cases}
\end{aligned}
$$

If follows that $N_{\lambda \mu}=\delta_{\bar{\lambda} \mu}$.

Remark 1.15. The isomorphism (1.14) is non-canonical. Actually, it doesn't even make sense to ask whether or not it is canonical since the right-hand side of the equation is only well defined up to non-canonical isomorphism.

Corollary 1.16. Let $S_{l}, S_{r}, S_{b}, S_{m}$ and $I_{1}, I_{2}, \ldots, I_{8}$ be as in (1.11). Then the algebra generated by $D\left(I_{4}\right)$ and $D\left(I_{6}\right)$ on $H_{\text {ann }}$ is canonically isomorphic to $\bigoplus_{\lambda \in \Delta_{D}} \mathbf{B}\left(H_{\lambda}\left(S_{m}\right.\right.$, D)). Moreover, there is a non-canonical isomorphism

$$
H_{\text {ann }} \bigotimes_{\left(D\left(I_{4}\right) \vee D\left(I_{6}\right)\right)^{\text {op }}} H_{0}\left(S_{m}, D\right) \cong H_{0}\left(S_{b}, D\right)
$$

which we represent as follows:

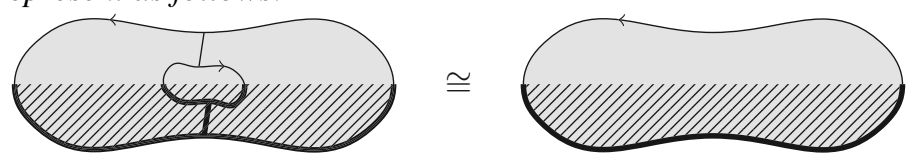

Proof. By the isomorphism (1.14), the Hilbert space $H_{a n n}$, when viewed as a representation of $D\left(I_{4}\right) \otimes_{\text {alg }} D\left(I_{6}\right)$, is a direct sum of copies of $H_{\lambda}\left(S_{m}\right)$, each one occurring with infinite multiplicity (more precisely with multiplicity $\left.\operatorname{dim}\left(H_{\bar{\lambda}}\left(S_{b}\right)\right)\right)$. Because each $H_{\lambda}\left(S_{m}\right)$ is irreducible as a representation of $D\left(I_{4}\right) \otimes_{\text {alg }} D\left(I_{6}\right)$, the von Neumann algebra generated by $D\left(I_{4}\right)$ and $D\left(I_{6}\right)$ on $H_{a n n}$ is a direct sum of type $I$ factors. The isomorphism (1.17) follows from the decomposition (1.14) because $H_{\lambda}\left(S_{m}\right) \otimes_{\left(D\left(I_{4}\right) \vee D\left(I_{6}\right)\right)^{\text {op }}} H_{0}\left(S_{m}, D\right) \cong \delta_{\lambda, 0} \mathbb{C}$. 
1.C. Extending defects to bicolored 1-manifolds. In [BDH17, Theorem 1.3], we extended the domain of definition of a conformal net from the category of intervals to the category of all compact 1-manifolds (where the morphisms are embeddings that are either orientation preserving or orientation reversing). In [BDH19, Eq. 1.34], we extended a defect to take values on disjoint unions of intervals. We now further extend a defect to all compact bicolored 1-manifolds, with an arbitrary number of color-change points. This extension will be useful when we construct the unit and counit sectors for adjunctions of defects, because the composite of a defect and its adjoint can be naturally reexpressed as the value of the defect on an interval with two color-change points.

Definition 1.18. A bicolored 1-manifold is a compact 1-manifold $M$ (always oriented), possibly with boundary, equipped with two compact submanifolds $M_{\circ}, M_{\bullet} \subset M$ such that $M_{\circ} \cap M_{\bullet}$ consists of finitely many points. Moreover, each point of $M_{\circ} \cap M_{\bullet}$ should be equipped with a local coordinate $(-\varepsilon, \varepsilon) \hookrightarrow M$ that sends $(-\varepsilon, 0]$ to $M_{\circ}$ and $[0, \varepsilon)$ to $M_{\bullet}$.

Given a bicolored 1-manifold $M$, we pick a decomposition $M=M_{0} \cup M_{1}$ such that $P:=M_{0} \cap M_{1}$ has finitely many points, none of which is a color-change point. Every connected component of $M_{0}$ and $M_{1}$ should be an interval, and should contain at most one color-change point. Pick local coordinates around $P$, and define $N_{i}:=\left(M_{i} \times\{1\}\right) \cup Q \subset$ $M \times[0,1]$, where $Q:=P \times[0,1]$ inherits its bicoloring from $P$. The manifolds $N_{i}$ and $Q$ are oriented so as to make the inclusions $M_{i} \rightarrow N_{i}$ and $Q \rightarrow N_{1}$ orientation preserving; the inclusion $Q \rightarrow N_{0}$ is then orientation reversing. The local coordinates around $P$ induce a smooth structure on $N_{i}$. As in [BDH19, Eq. 1.34], we define the defect on a disjoint union of bicolored intervals by $D\left(I_{1} \cup \cdots \cup I_{n}\right):=D\left(I_{1}\right) \bar{\otimes} \cdots \bar{\otimes} D\left(I_{n}\right)$. We then define the defect on any bicolored 1-manifold as follows.

Definition 1.19. Given a defect $D$ and a bicolored 1-manifold $M$, we define the value of $D$ on $M$ to be

$$
D(M):=D\left(N_{0}\right) \circledast D(Q) D\left(N_{1}\right) .
$$

(See [BDH19, Section 1.E \& Appendix B.IV] for discussion and the definition of the relative fusion product $\circledast$ of von Neumann algebras.)

In [BDH17, Corollary 1.13], we showed that the value of a conformal net on a 1manifold was independent of the choice of decomposition used in the definition; the same argument generalizes to the situation here, showing that the algebra (1.20) is independent (up to canonical isomorphism) of the choice of decomposition $M=M_{1} \cup M_{2}$.

Here is an example of the above definition:

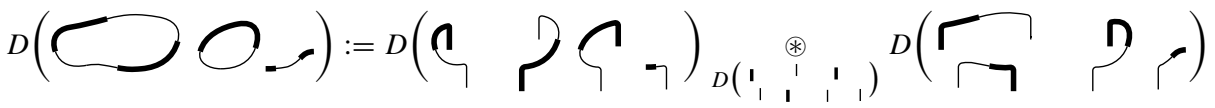

In Sect. 2.C, this extension of a defect to take values on all bicolored 1-manifolds will allow a computationally convenient expression for the composite of a defect and its dual.

Proposition 1.21. Let ${ }_{\mathcal{A}} D_{\mathcal{B}}$ be a finite defect. Let $S^{1}$ be the standard bicolored circle, let $I \subset S^{1}$ be the bicolored 1-dimensional submanifold indicated in the following picture

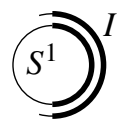

and let $D(I)$ be as in Definition 1.19. Then the natural action of $D\left(I \cap S_{\top}^{1}\right) \otimes_{\text {alg }} D\left(I \cap S_{\perp}^{1}\right)$ on $H_{0}(D)$ extends to a normal (that is, ultraweakly continuous) action of $D(I)$. 
Proof. We first address the case when $D$ is irreducible. By definition, the algebra $D(I)$ acts (normally) on

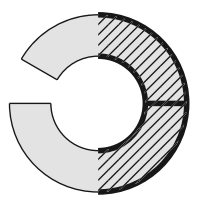

Fusing in $\bigcirc$, we can use the fact that a vacuum sector of a conformal net fuses with a vacuum sector of a defect to a vacuum sector of the defect [BDH19, Lemma 1.15] and the fact that cyclic fusion is cyclically invariant [BDH17, Appendix A] to see that $D(I)$ also acts on

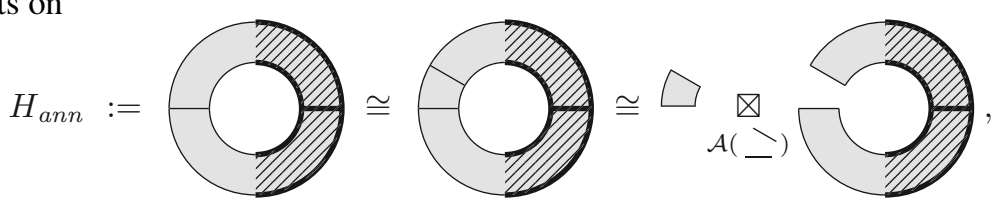

where the second picture denotes the cyclic fusion of $\triangle$,

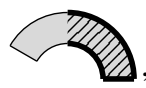
and

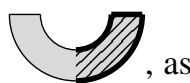
defined in [BDH17, Appendix A].

By Corollary 1.16, the algebra generated by $D(\neg)$ and $D(\curlywedge)$ in $\mathbf{B}\left(H_{a n n}\right)$ admits a natural right action on Since the action of $D(I)$ on $H_{\text {ann }}$ commutes with that of $D(\neg) \vee D(\smile)$, the algebra $D(I)$ also acts on

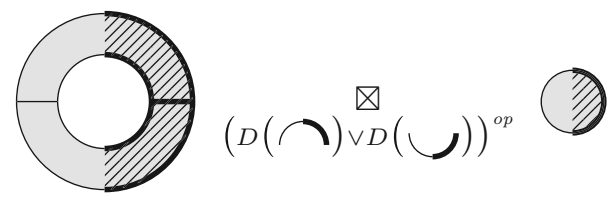

By (1.17), the latter is isomorphic to $H_{0}(D)$.

When $D$ is not irreducible, write it as a sum $D_{1} \oplus \cdots \oplus D_{n}$ of irreducible defects. We then have $H_{0}(D)=\bigoplus_{i} H_{0}\left(D_{i}\right)$, and

$$
\begin{aligned}
D(I)=D(\supset) & =D(\supset)_{\mathcal{B}(-)}^{\circledast} D(\text { J }) \\
& =\bigoplus_{i, j} D_{i}(\boldsymbol{J})_{\mathcal{B}(-)}^{\circledast} D_{j}(\text { ᄀ })
\end{aligned}
$$

The subalgebra $D_{i}\left(I \cap S_{\text {个 }}^{1}\right) \otimes_{\text {alg }} D_{j}\left(I \cap S_{\perp}^{1}\right) \subset D(I)$ acts as zero on $H_{0}\left(D_{k}\right)$ unless $i=j=k$, in which case the first part of the proof applies and it extends to a normal action of $D_{i}(I)$ on $H_{0}\left(D_{i}\right)$. Thus the action of $D\left(I \cap S_{\top}^{1}\right) \otimes_{\text {alg }} D\left(I \cap S_{\perp}^{1}\right)=\bigoplus_{i, j} D_{i}(I \cap$ $\left.S_{\text {个 }}^{1}\right) \otimes_{\text {alg }} D_{j}\left(I \cap S_{\perp}^{1}\right)$ on $\bigoplus H_{0}\left(D_{i}\right)$ extends to a normal action of $D(I)$. 


\section{A Characterization of Dualizable Conformal Nets}

2.A. Involutions on nets, defects, sectors, and intertwiners. The 3-category $\mathrm{CN}$ is equipped with four antilinear involutions ${ }^{*},{ }^{-},{ }^{\dagger},{ }^{o p}$, where the $i$ th involution is contravariant at the level of $(4-i)$-morphisms, and covariant at all other levels. The second and third involutions will provide adjoints for finite sectors and defects respectively, and the fourth involution will provide the dual of a conformal net-that the involutions do indeed give adjoints, respectively duals, is proven in Sect. 2.C.

The first involution * acts trivially on the 0,1 , and 2-morphisms, and sends a 3 morphism $f: H \rightarrow K$ to its adjoint $f^{*}: K \rightarrow H$ (in the sense of maps between Hilbert spaces).

The second one ${ }^{-}$acts trivially on 0 and on 1 -morphisms. It sends a $D$ - $E$-sector $\left(H,\left\{\rho_{I}\right\}\right)$, where the homomorphisms $\rho_{I}$ are given by

$$
\begin{array}{llll}
\rho_{I}: \mathcal{A}(I) \rightarrow \mathbf{B}(H) & \text { for } \quad I \in \mathrm{INT}_{S^{1}, \circ} & \rho_{I}: D(I) \rightarrow \mathbf{B}(H) & \text { for } I \in \mathrm{INT}_{S^{1}, \top} \\
\rho_{I}: \mathcal{B}(I) \rightarrow \mathbf{B}(H) & \text { for } I \in \mathrm{INT}_{S^{1}, \bullet} & \rho_{I}: E(I) \rightarrow \mathbf{B}(H) & \text { for } I \in \mathrm{INT}_{S^{1}, \perp}
\end{array}
$$

to the complex conjugate Hilbert space $\bar{H}$ and $E$-D-sector structure given by

$$
\begin{array}{ll}
\bar{\rho}_{I}: \mathcal{A}(I) \rightarrow \mathbf{B}(\bar{H}) \text { for } I \in \mathrm{INT}_{S^{1}, \circ} & \bar{\rho}_{I}: E(I) \rightarrow \mathbf{B}(\bar{H}) \text { for } I \in \mathrm{INT}_{S^{1}, \top} \\
\bar{\rho}_{I}: \mathcal{B}(I) \rightarrow \mathbf{B}(\bar{H}) \text { for } I \in \mathrm{INT}_{S^{1}, \bullet} & \bar{\rho}_{I}: D(I) \rightarrow \mathbf{B}(\bar{H}) \text { for } I \in \mathrm{INT}_{S^{1}, \perp}
\end{array}
$$

where $\bar{\rho}_{I}(a):=\rho_{j(I)}\left(j_{*}\left(a^{*}\right)\right)$, and $j: z \mapsto \bar{z}$ is the reflection in the horizontal axis. Here, $j_{*}$ stands for either $\mathcal{A}(j), E(j), \mathcal{B}(j)$, or $D(j)$. The involution - sends a 3-morphism $f: H \rightarrow K$ to its complex conjugate $\bar{f}: \bar{H} \rightarrow \bar{K}$.

The third involution $\dagger$ acts trivially on objects. Given a bicolored interval $I$, let $I^{\text {rev }}$ denote the same interval with reversed bicoloring, that is, $\left(I^{\mathrm{rev}}\right)_{\circ}=I_{\bullet}$ and $\left(I^{\mathrm{rev}}\right)_{\bullet}=I_{\circ}$. The orientation of $I^{\mathrm{rev}}$ is the same as that of $I$, but the local coordinate is negated. The reversed defect of ${ }_{\mathcal{A}} D_{\mathcal{B}}$ is the defect ${ }_{\mathcal{B}} D^{\dagger} \mathcal{A}$ defined by $D^{\dagger}(I)=D\left(I^{\text {rev }}\right)$. For a $D$-E-sector $H$, the corresponding $D^{\dagger}-E^{\dagger}$-sector $H^{\dagger}$ is the complex conjugate of $H$, with structure maps

$$
\begin{array}{llll}
\rho_{I}^{\dagger}: \mathcal{B}(I) & \rightarrow \mathbf{B}\left(H^{\dagger}\right) \text { for } I \in \mathrm{INT}_{S^{1}, \circ} & \rho_{I}^{\dagger}: D^{\dagger}(I) \rightarrow \mathbf{B}\left(H^{\dagger}\right) & \text { for } I \in \mathrm{INT}_{S^{1}, \top} \\
\rho_{I}^{\dagger}: \mathcal{A}(I) & \rightarrow \mathbf{B}\left(H^{\dagger}\right) \text { for } I \in \mathrm{INT}_{S^{1}, \bullet} & \rho_{I}^{\dagger}: E^{\dagger}(I) \rightarrow \mathbf{B}\left(H^{\dagger}\right) & \text { for } I \in \mathrm{INT}_{S^{1}, \perp}
\end{array}
$$

given by $\rho_{I}^{\dagger}(a)=\rho_{r(I)}\left(r_{*}\left(a^{*}\right)\right)$, where $r: z \mapsto-\bar{z}$ is now the vertical reflection. 3-morphisms are sent to their complex conjugates.

The fourth involution ${ }^{o p}$ sends $\mathcal{A} \in \mathrm{CN}$ to the a conformal net $\mathcal{A}^{o p}(I):=\mathcal{A}(I)^{o p}$. Similarly, it sends a morphism ${ }_{\mathcal{A}} D_{\mathcal{B}}$ to the $\mathcal{A}^{o p}-\mathcal{B}^{o p}$-defect $D^{o p}(I):=D(I)^{o p}$. A $D-E$ sector $\left(H,\left\{\rho_{I}\right\}\right)$ is sent to the complex conjugate Hilbert space, with actions $\rho_{I}^{o p}\left(a^{o p}\right):=$ $\rho_{I}\left(a^{*}\right)$. Finally, 3-morphisms go to their complex conjugates.

Remark 2.2. The existence of these four involutions ensures that any duality or adjunction in $\mathrm{CN}$ is automatically ambidextrous, that is, it is both a left and a right duality or adjunction. (When we say ' $X$ has ambidextrous adjoint (or dual) $Y$ ', we mean that $Y$ admits both the structure of a left and the structure of a right adjoint (or dual) to $X$.) 
2.B. The snake interchange isomorphism for defects. To establish, in the next section, that the reversed defect ${ }_{\mathcal{B}} D_{\mathcal{A}}^{\dagger}$ is an (ambidextrous) adjoint of the $\operatorname{defect}{ }_{\mathcal{A}} D_{\mathcal{B}}$, we will need the following variant of the sector interchange isomorphism [BDH19, Eq. 6.25].

To simplify the maneuvers involved in this interchange isomorphism, here and for the remainder of the paper, we use a model for the vertical composition of sectors that fuses sectors along one-quarter of their boundary:

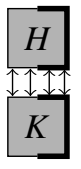

This is by contrast with the model we used previously, in [BDH19], which involved fusing along half of the boundary of each sector. The equivalence between these two fusions is discussed in Appendix B.

Let $\mathcal{A}, \mathcal{B}, \mathcal{C}$ be conformal nets, let ${ }_{\mathcal{A}} D_{\mathcal{B}},{ }_{\mathcal{B}} E_{\mathcal{C}},{ }_{\mathcal{B}} F_{\mathcal{C}},{ }_{\mathcal{A}} G_{\mathcal{C}}$ be defects, let $H$ be an $F$ $E$-sector, and let $K$ be a $D \circledast \mathcal{B} E-G$-sector. We are interested in two ways of evaluating the diagram

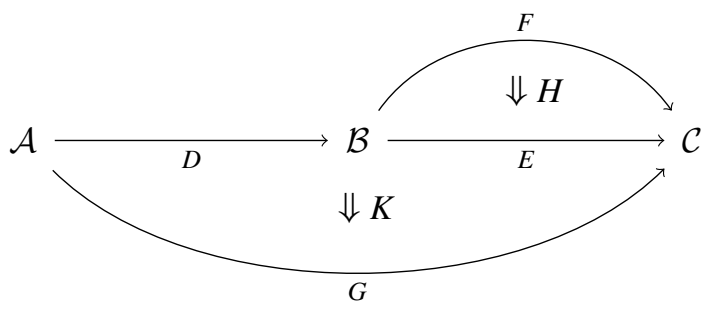

i.e., of fusing the three sectors

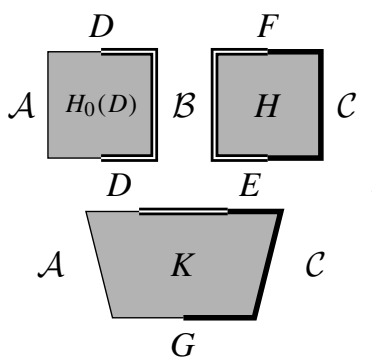

Let us name and orient the relevant intervals $I_{1}, I_{2}, \ldots, I_{10}$ as indicated here:

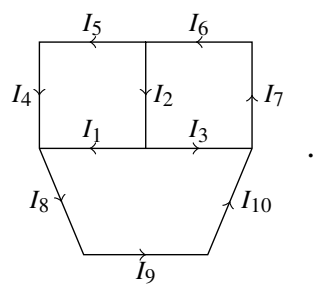

All of them are copies of the standard interval [0, 1]. Let also $S_{l}:=\bar{I}_{1} \cup \bar{I}_{2} \cup I_{5} \cup I_{4}$, $S_{r}:=I_{2} \cup I_{3} \cup I_{7} \cup I_{6}, S_{b}:=I_{8} \cup I_{9} \cup I_{10} \cup \bar{I}_{3} \cup I_{1}, S_{l r}:=\bar{I}_{1} \cup I_{3} \cup I_{7} \cup I_{6} \cup I_{5} \cup I_{4}$, $S_{l b}:=I_{8} \cup I_{9} \cup I_{10} \cup \bar{I}_{3} \cup \bar{I}_{2} \cup I_{5} \cup I_{4}$, and $S_{l r b}:=I_{8} \cup I_{9} \cup I_{10} \cup I_{7} \cup I_{6} \cup I_{5} \cup I_{4}$, where we have used bars to indicate reverse orientation. 
Lemma 2.4. There is a non-canonical unitary isomorphism

$$
\left(H_{0}\left(S_{l}, D\right) \bigotimes_{D\left(I_{1}\right)} K\right) \underset{\mathcal{B}\left(I_{2}\right) \vee E\left(I_{3}\right)}{\bigotimes} H \cong\left(H_{0}\left(S_{l}, D\right) \bigotimes_{\mathcal{B}\left(I_{2}\right)} H\right) \underset{D\left(I_{1}\right) \vee E\left(\bar{I}_{3}\right)}{\otimes} K,
$$

equivariant with respect to $\mathcal{A}\left(I_{4}\right), D\left(I_{5}\right), E\left(I_{6}\right), \mathcal{C}\left(I_{7}\right), \mathcal{A}\left(I_{8}\right), G\left(I_{9}\right)$, and $\mathcal{C}\left(I_{10}\right)$.

Proof. For fixed $\mathcal{A}, \mathcal{B}, \mathcal{C}, D, E, G, K$, the desired isomorphism (2.5) can be thought of as a natural transformation

$$
E\left(I_{2} \cup I_{3}\right) \text {-modules 业 } D\left(I_{4} \cup I_{5}\right) \otimes_{\text {alg }} G\left(I_{8} \cup I_{9} \cup I_{10}\right) \text {-modules }
$$

between functors of the variable $H$. The fact that (2.5) commutes with the action of $F\left(I_{6} \cup I_{7}\right)$ is then encoded in the naturality of (2.6).

Since $H_{0}(E)$ is a faithful $E\left(I_{2} \cup I_{3}\right)$-module, it is enough, by [BDH19, Lemma B.24], to construct the isomorphism (2.5) for $H=H_{0}(E)$ and check that it commutes with the action of $F\left(I_{6} \cup I_{7}\right)$. Pick involutions $\varphi \in \operatorname{Diff}_{-}\left(S_{l}\right), \psi \in \operatorname{Diff}_{-}\left(S_{r}\right), \chi \in \operatorname{Diff}_{-}\left(S_{l r}\right)$ such that

$$
\varphi\left(I_{4} \cup I_{5} \cup I_{2}\right)=I_{1}, \quad \psi\left(I_{6} \cup I_{7}\right)=I_{2} \cup I_{3}, \quad \chi\left(I_{4} \cup I_{5} \cup I_{6} \cup I_{7}\right)=I_{1} \cup I_{3},
$$

and corresponding (non-canonical) unitaries $u: H_{0}(D) \stackrel{\cong}{\rightarrow} L^{2}\left(D\left(I_{1}\right)\right), v: H_{0}(E) \stackrel{\cong}{\rightrightarrows}$ $L^{2}\left(E\left(I_{2} \cup I_{3}\right)\right)$, and $w: H_{0}(D \circledast \mathcal{B} E) \stackrel{\cong}{\rightarrow} L^{2}\left(D\left(I_{1}\right) \vee E\left(I_{3}\right)\right)$, as in [BDH19, Lemma 1.13]. Let also

$$
\begin{aligned}
\alpha & :=\left.\varphi\right|_{I_{4} \cup I_{5} \cup I_{2}} \cup \operatorname{Id}_{I_{8} \cup I_{9} \cup I_{10} \cup I_{3}}: S_{l b} \rightarrow S_{b}, \\
\beta & :=\left.\psi\right|_{I_{6} \cup I_{7}} \cup \operatorname{Id}_{I_{5} \cup I_{4} \cup I_{8} \cup I_{9} \cup I_{10}}: S_{l r b} \rightarrow S_{l b}, \\
\gamma & :=\left.\chi\right|_{I_{4} \cup I_{5} \cup I_{6} \cup I_{7}} \cup \operatorname{Id}_{I_{8} \cup I_{9} \cup I_{10}}: S_{l r b} \rightarrow S_{b} .
\end{aligned}
$$

We may assume that $\varphi, \psi$, and $\chi$ are chosen so that $\alpha \circ \beta=\gamma$. The isomorphism (2.5) for $H=H_{0}(E)$ can then be written explicitly:

$$
\begin{aligned}
& \left(H_{0}(D) \bigotimes_{D\left(I_{1}\right)} K\right) \bigotimes_{E\left(I_{2} \cup I_{3}\right)} H_{0}(E) \stackrel{u \otimes 1}{\longrightarrow}\left(L^{2}\left(D\left(I_{1}\right)\right) \bigotimes_{D\left(I_{1}\right)} K\right) \bigotimes_{E\left(I_{2} \cup I_{3}\right)} H_{0}(E) \\
& \stackrel{\cong}{\rightrightarrows} \alpha^{*} K \otimes_{E\left(I_{2} \cup I_{3}\right)} H_{0}(E) \stackrel{1 \otimes v}{\longrightarrow} \alpha^{*} K \bigotimes_{E\left(I_{2} \cup I_{3}\right)} L^{2}\left(E\left(I_{2} \cup I_{3}\right)\right) \\
& \stackrel{\cong}{\rightarrow} \beta^{*} \alpha^{*} K=\gamma^{*} K \stackrel{\cong}{\rightarrow} L^{2}\left(D\left(I_{1}\right) \vee E\left(\bar{I}_{3}\right)\right) \otimes_{D\left(I_{1}\right) \vee E\left(\bar{I}_{3}\right)} K \\
& \stackrel{w^{-1} \otimes 1}{\longrightarrow} H_{0}(D \circledast \mathcal{B} E) \otimes_{D\left(I_{1}\right) \vee E\left(\bar{I}_{3}\right)} K \stackrel{\Omega \otimes 1}{\longrightarrow}\left(H_{0}(D) \bigotimes_{\mathcal{B}\left(I_{2}\right)} H_{0}(E)\right) \bigotimes_{D\left(I_{1}\right) \vee E\left(\bar{I}_{3}\right)} K,
\end{aligned}
$$

where $\Omega$ denotes the " $1 \otimes 1$-isomorphism” constructed in [BDH19, Theorem 6.2].

Generalizing (2.3), we now consider this situation:

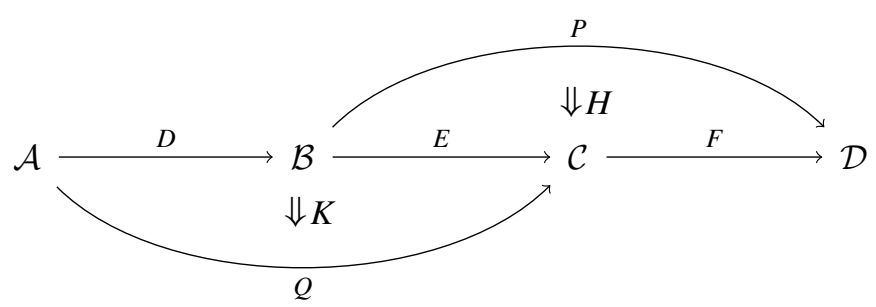


which corresponds (using Appendix B) to the following configuration of sectors:

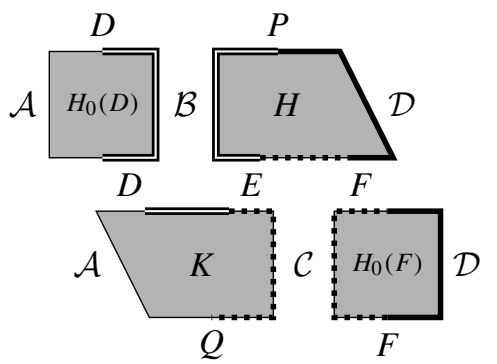

We name the relevant intervals $I_{1}, I_{2}, \ldots, I_{13}$ :

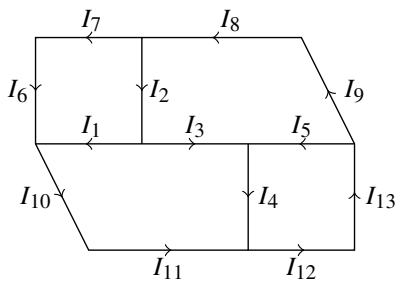

Once again, all these intervals are copies of the standard interval $[0,1]$.

Lemma 2.9. Let $\mathcal{A}, \mathcal{B}, \mathcal{C}, \ldots, K$ be as in (2.7). Then there is a non-canonical unitary isomorphism

$$
\begin{aligned}
H_{0}(D) \underset{D\left(I_{1}\right) \vee \mathcal{B}\left(I_{2}\right)}{\otimes}\left(K \otimes_{E\left(I_{3}\right)} H\right) \underset{\mathcal{C}\left(I_{4}\right) \vee F\left(I_{5}\right)}{\otimes} H_{0}(F) \\
\quad \cong\left(H_{0}(D) \otimes_{\mathcal{B}\left(I_{2}\right)} H\right) \underset{D\left(I_{1}\right) \vee E\left(I_{3}\right) \vee F\left(I_{5}\right)}{\otimes}\left(K \otimes_{\mathcal{C}\left(I_{4}\right)} H_{0}(F)\right)
\end{aligned}
$$

that is equivariant with respect to the actions of the algebras $\mathcal{A}\left(I_{6}\right), D\left(I_{7}\right), P\left(I_{8}\right), \mathcal{D}\left(I_{9}\right)$, $\mathcal{A}\left(I_{10}\right), Q\left(I_{11}\right), F\left(I_{12}\right)$, and $\mathcal{D}\left(I_{13}\right)$.

Proof. Fix $\mathcal{A}, \mathcal{B}, \mathcal{C}, \mathcal{D}, D, E, F$. We shall construct a natural transformation

$$
\begin{aligned}
\left(E \circledast_{\mathcal{C}} F\right)\left(L_{-}\right) \text {-modules } & \times\left(D \circledast_{\mathcal{B}} E\right)(\rightarrow) \text {-modules } \\
& \mathbb{\Psi}_{>}^{*} D\left(I_{7} \cup I_{6}\right) \otimes_{\text {alg }} F\left(I_{12} \cup I_{13}\right) \text {-modules, }
\end{aligned}
$$

where $(E \circledast \mathcal{C} F)\left(L_{\zeta_{-}}\right)=E\left(I_{2} \cup I_{3}\right) \vee F\left(\bar{I}_{5}\right)$ and $\left.(D \circledast \mathcal{B} E)(-\rceil\right)=D\left(I_{1}\right) \vee E\left(\bar{I}_{3} \cup \bar{I}_{4}\right)$, as in [BDH19, Definition 1.43]. The isomorphism (2.10) is the value of that natural transformation on the object $(H, K)$.

By [BDH19, Lemma B.24], it is enough to construct the above natural transformation for the pair $\left(H=H_{0}(E \circledast \mathcal{C} F), K=H_{0}(D \circledast \mathcal{B} E)\right)$. In that case, it is given by

$$
\begin{gathered}
H_{0}(D) \underset{D\left(I_{1}\right) \vee \mathcal{B}\left(I_{2}\right)}{\otimes}\left(H_{0}(D \circledast \mathcal{B} E) \underset{E\left(I_{3}\right)}{\otimes} H_{0}(E \circledast \mathcal{C} F)\right) \underset{\mathcal{C}\left(I_{4}\right) \vee F\left(I_{5}\right)}{\otimes} H_{0}(F) \\
\stackrel{1 \otimes 1 \otimes \Omega \otimes 1}{\longrightarrow} \\
H_{0}(D) \underset{D\left(I_{1}\right) \vee \mathcal{B}\left(I_{2}\right)}{\otimes}\left(H_{0}(D \circledast \mathcal{B} E) \underset{E\left(I_{3}\right)}{\otimes} H_{0}(E) \underset{\mathcal{C}}{\otimes} H_{0}(F)\right) \underset{\mathcal{C}\left(I_{4}\right) \vee F\left(I_{5}\right)}{\otimes} H_{0}(F)
\end{gathered}
$$




$$
\begin{aligned}
& \cong\left(H_{0}(D) \underset{D\left(I_{1}\right) \vee \mathcal{B}\left(I_{2}\right)}{\otimes}\left(H_{0}(D \circledast \mathcal{B} E) \underset{E\left(I_{3}\right)}{\otimes} H_{0}(E)\right)\right) \underset{\mathcal{C}}{\otimes} H_{0}(F) \underset{\mathcal{C}\left(I_{4}\right) \vee F\left(I_{5}\right)}{\otimes} H_{0}(F) \\
& \text { Lemma } 2.4 \otimes 1 \otimes 1 \\
& \left(\left(H_{0}(D) \underset{\mathcal{B}\left(I_{2}\right)}{\otimes} H_{0}(E)\right) \underset{D\left(I_{1}\right) \vee E\left(\bar{I}_{3}\right)}{\otimes} H_{0}(D \circledast \mathcal{B} E)\right) \underset{\mathcal{C}}{\otimes} H_{0}(F) \underset{\mathcal{C}\left(I_{4}\right) \vee F\left(I_{5}\right)}{\otimes} H_{0}(F) \\
& \cong\left(\left(H_{0}(D) \underset{\mathcal{B}\left(I_{2}\right)}{\otimes} H_{0}(E) \underset{\mathcal{C}}{\otimes} H_{0}(F)\right) \underset{D\left(I_{1}\right) \vee E\left(I_{3}\right)}{\otimes} H_{0}(D \circledast \mathcal{B} E)\right) \underset{\mathcal{C}\left(I_{4}\right) \vee F\left(I_{5}\right)}{\otimes} H_{0}(F) \\
& \stackrel{1 \otimes \Omega^{-1} \otimes 1 \otimes 1}{\longrightarrow} \\
& \cong\left(\left(H_{0}(D) \underset{\mathcal{B}\left(I_{2}\right)}{\otimes} H_{0}(E \circledast \mathcal{C} F)\right) \underset{D\left(I_{1}\right) \vee E\left(\bar{I}_{3}\right)}{\otimes} H_{0}(D \circledast \mathcal{B} E)\right) \underset{\mathcal{C}\left(I_{4}\right) \vee F\left(I_{5}\right)}{\otimes} H_{0}(F) \\
& \stackrel{\text { Lemma } 2.4}{\longrightarrow} \\
& \left(H_{0}(D) \underset{\mathcal{B}\left(I_{2}\right)}{\bigotimes} H_{0}(E \circledast \mathcal{C} F)\right) \underset{D\left(I_{1}\right) \vee E\left(I_{3}\right) \vee F\left(I_{5}\right)}{\bigotimes}\left(H_{0}(D \circledast \mathcal{B} E) \underset{\mathcal{C}\left(I_{4}\right)}{\otimes} H_{0}(F)\right) .
\end{aligned}
$$

2.C. Finite nets are dualizable. We investigate the relationship of finiteness and dualizability for, in turn, sectors, defects, and nets.

Dualizability for sectors. Recall that all defects are assumed to be semisimple.

Proposition 2.11. A sector ${ }_{D} H_{E}$ has an adjoint (necessarily ambidextrous) if and only if it is finite. In this case, the adjoint is canonically isomorphic to ${ }_{E} \bar{H}_{D}$.

Proof. If the sector ${ }_{D} H_{E}$ has an adjoint ${ }_{E} K_{D}$, that adjoint sector provides the (am-

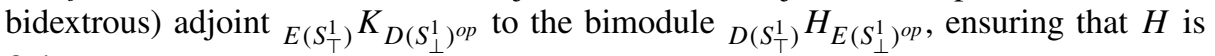
finite.

Conversely, if $H$ is a dualizable $D\left(S_{\mathrm{T}}^{1}\right)-E\left(S_{\perp}^{1}\right)^{o p}$-bimodule then, by [BDH14, Corollary 6.12] and the fact that $D$ and $E$ are semisimple, its dual is canonically isomorphic to $\bar{H}$, with the $E\left(S_{\perp}^{1}\right)^{o p}-D\left(S_{\top}^{1}\right)$-bimodule structure given by $a \bar{\xi} b=\overline{b^{*} \xi a^{*}}$. Identify the left action of $E\left(S_{\perp}^{1}\right)^{o p}$ with a left action of $E\left(S_{\top}^{1}\right)$, and the right action of $D\left(S_{\top}^{1}\right)$ with a left action of $D\left(S_{\perp}^{1}\right)$ via the isomorphisms $j_{*}: E\left(S_{\perp}^{1}\right)^{o p} \rightarrow E\left(S_{\top}^{1}\right)$ and $j_{*}: D\left(S_{\top}^{1}\right)^{o p} \rightarrow D\left(S_{\perp}^{1}\right)$; then extend these actions to the structure of an $E$ - $D$-sector on $\bar{H}$ according to (2.1). The unit and counit bimodule intertwiners for the bimodule duality serve, in fact, as sector intertwiners, providing ${ }_{E} \bar{H}_{D}$ with the structure of an adjoint sector to ${ }_{D} H_{E}$.

By Remark 2.2, we have the following:

Corollary 2.12. A sector is dualizable if and only if it is finite.

Dualizability for defects. Given a bicolored interval $I$, we define the following two bicolored manifolds $\bar{I}$ and $\bar{I}$. The underlying manifold of $\bar{I}$ and of $\bar{I}$ are both given by $I_{\circ} \cup[0,1] \cup I_{\bullet}$, and their bicolorings are

$$
\bar{I}_{\circ}=[0,1], \quad \bar{I}_{\bullet}=I_{\circ} \cup I_{\bullet}, \quad \bar{I}_{\circ}=I_{\circ} \cup I_{\bullet}, \quad \bar{I}_{\bullet}=[0,1] .
$$


Here is an example illustrating the above concepts:
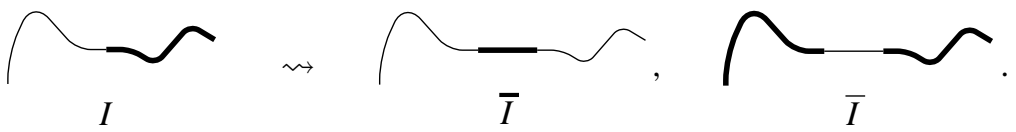

Let $D$ be an $\mathcal{A}$ - $\mathcal{B}$-defect. Definition 1.19 is made so as to provide an easy description of $D \circledast D^{\dagger}$ and $D^{\dagger} \circledast D$. They are given by

$$
\left(D \circledast \mathcal{B} D^{\dagger}\right)(I)=D(\bar{I}) \quad \text { and } \quad\left(D^{\dagger} \circledast \mathcal{A} D\right)(I)=D(\bar{I}),
$$

essentially by definition.

Proposition 2.14. Let $\mathcal{A}$ and $\mathcal{B}$ be finite conformal nets. Every finite defect ${ }_{\mathcal{A}} D_{\mathcal{B}}$ has ambidextrous adjoint ${ }_{\mathcal{B}} D_{\mathcal{A}}^{\dagger}$, and the unit and counit sectors of both the left and right adjunctions are finite.

Proof. By Remark 2.2, it suffices to consider just one of the two adjunctions.

Let $\bar{S}^{1}$ be the bicolored manifold obtained by taking the standard circle $S^{1}$, cutting it open at $i \in S^{1}$, and then glueing in a copy of $[0,1]$. The black part of $\bar{S}^{1}$ is the interval $[0,1]$ that is added on the top, and all the rest is white. Similarly, let $\underline{S}^{1}$ be the bicolored manifold that is obtained by inserting a white interval at the location of $-i \in S^{1}$, and coloring all the rest black.
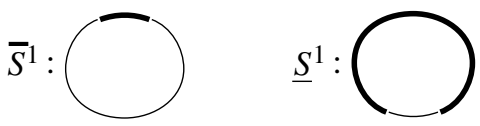

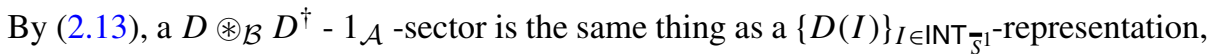
where INT $\bar{S}^{1}$ denotes the poset of subintervals $I \subset \bar{S}^{1}, \partial I \cap \bar{S}_{\bullet}^{1}=\emptyset$, that are allowed to contain $\bar{S}_{\bullet}^{1}$ in their interior, but that are not allowed to contain $\bar{S}_{\circ}^{1}$. Pick a color preserving diffeomorphism $\varphi$ from $\bar{S}^{1}$ to the standard bicolored circle. By Proposition 1.21, we can

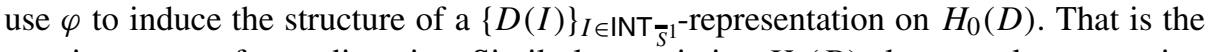
counit sector $r$ of our adjunction. Similarly, restricting $H_{0}(D)$ along a color preserving diffeomorphism from $\underline{S}$ to the standard bicolored circle provides a $1_{\mathcal{B}}-D^{\dagger} \circledast \mathcal{A} D$-sector $s$, which is the unit of our adjunction. The sectors $r$ and $s$ are finite by the finiteness of any defect vacuum sector with respect to these boundary decompositions [BDH19, Lemma 3.17]. 5

We now have to show that $r$ and $s$ satisfy the duality equations

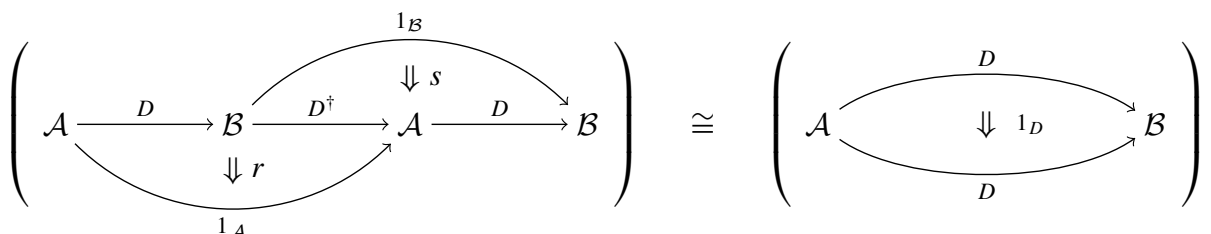

5 This vacuum sector finiteness result [BDH19, Lemma 3.17] was stated for irreducible defects, but it also holds for semisimple defects and in fact for arbitrary defects, using the direct integral decomposition [BDH19, Lemma 1.32]. 
and

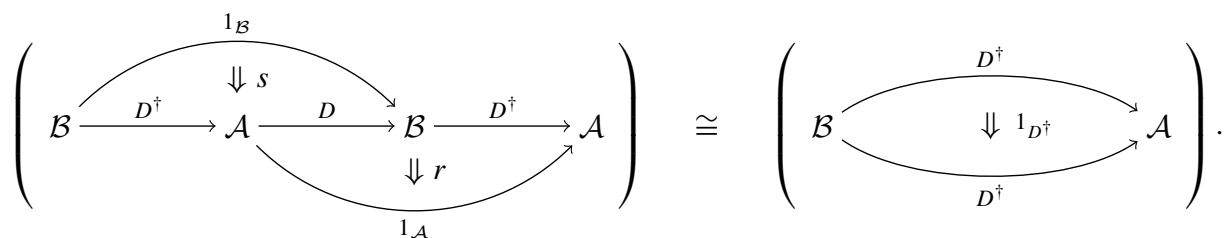

We only check the first equation, the second one being completely analogous. Let $I_{1}, \ldots, I_{13}$ be as in (2.8). By Lemma 2.9 and Appendix B, the left-hand side $\mathcal{A} \longrightarrow \mathcal{B} \longrightarrow \mathcal{\Downarrow} \longrightarrow \mathcal{B}$ is isomorphic to

$$
H_{0}(D) \underset{D\left(I_{1} \cup I_{2}\right)}{\bigotimes}\left(r \underset{D^{\dagger}\left(I_{3}\right)}{\bigotimes} s\right) \underset{D\left(I_{4} \cup I_{5}\right)}{\otimes} H_{0}(D) .
$$

Because the fusion of two vacuum sectors for a defect is again a vacuum sector for that same defect [BDH19, Lemma 1.15], the middle term $r \bigotimes_{D^{\dagger}\left(I_{3}\right)} s$ is the vacuum sector of $D$ associated to $I_{1} \cup I_{10} \cup I_{11} \cup \bar{I}_{4} \cup \bar{I}_{5} \cup I_{9} \cup I_{8} \cup I_{2}$. By two more applications of that same lemma, we identify (2.15) with the identity sector on $D$.

Recall that all conformal nets and defects are assumed to be semisimple. Combining the above proposition with Corollary 2.12, we have the following.

Corollary 2.16. Every finite defect between finite conformal nets is fully dualizable.

Dualizability for conformal nets. In [BDH18], we constructed a 3-category whose objects are finite conformal nets, whose morphisms are defects, whose 2-morphisms are sectors, and whose 3 -morphisms are intertwiners. ${ }^{6}$ If $\mathcal{A}$ and $\mathcal{B}$ are conformal nets that are notnecessarily finite, then, even though we do not know that they live in a 3-category, we can still make sense of $\mathcal{A}$ and $\mathcal{B}$ being dual: specifically, $\mathcal{B}$ is the left dual of $\mathcal{A}$ if there

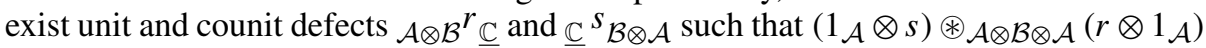
and $\left(s \otimes 1_{\mathcal{B}}\right) \circledast \mathcal{B} \otimes \mathcal{A} \otimes \mathcal{B}\left(1_{\mathcal{B}} \otimes r\right)$ are defects, and are equivalent (in the 2 -category of $\mathcal{A}$ - $\mathcal{A}$-defects or $\mathcal{B}$ - $\mathcal{B}$-defects) to the identity defects on $\mathcal{A}$ and $\mathcal{B}$, respectively. (Note that by convention both the tensor product $\otimes$ and the relative fusion product $\circledast$ have a geometric, not functorial, composition order.)

Theorem 2.17. An arbitrary conformal net $\mathcal{A}$ has ambidextrous dual $\mathcal{A}^{\text {op }}$. If $\mathcal{A}$ is finite, then the unit and counit defects of both the left and right dualities are themselves finite.

Proof. By Remark 2.2, it is enough to discuss just one of the two dualities. We show that $\mathcal{A} \dashv \mathcal{A}^{\text {op }}$.

Given a bicolored interval, let $I_{\bullet}:=I_{\circ} \cap I_{\bullet}$. It consists of one point if $I$ is genuinely bicolored, and it is empty otherwise. The counit defect $\mathcal{A} \otimes \mathcal{A}^{o p}{ }_{\mathbb{C}}$ and the unit defect $\mathbb{C}^{S} \mathcal{A}^{o p} \otimes \mathcal{A}$ are defined by

$$
r: I \mapsto \mathcal{A}\left(I_{\circ} \cup_{I_{\infty}} \bar{I}_{\circ}\right) \quad \text { and } \quad s: I \mapsto \mathcal{A}\left(\bar{I}_{\bullet} \cup_{I_{\bullet}} I_{\bullet}\right)
$$

\footnotetext{
6 Insisting that the conformal nets be finite allowed us to prove that the composition of two defects is again a defect; we do not know if the composition of defects between arbitrary conformal nets is a defect, in particular whether the composite satisfies the vacuum sector axiom [BDH19, Definition 1.7, axiom (iv)].
} 
where the bar stands for orientation reversal. In pictures, this is:

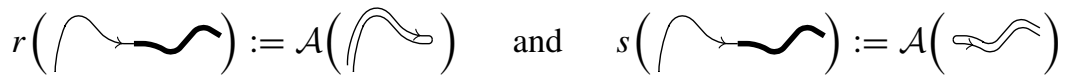

We now verify the two duality equations for $r$ and $s$. We need to show that the fusions $\left(1_{\mathcal{A}} \otimes s\right) \circledast \mathcal{A} \otimes \mathcal{A}^{o p} \otimes \mathcal{A}\left(r \otimes 1_{\mathcal{A}}\right)$ and $\left(s \otimes 1_{\mathcal{A}^{o p}}\right) \circledast \mathcal{A}^{o p} \otimes \mathcal{A} \otimes \mathcal{A}^{o p}\left(1_{\mathcal{A}^{o p}} \otimes r\right)$ are indeed defects, and are equivalent to identity defects on $\mathcal{A}$ and $\mathcal{A}^{o p}$, respectively. Let $I$ be a genuinely bicolored interval. By [BDH17, Lemma 1.12], the definition of the above fusions reduces to

$$
\begin{aligned}
& ((1 \otimes s) \circledast(r \otimes 1))(I)=\mathcal{A}\left(I_{\circ} \cup_{\{0\}}[0,1] \cup_{\{1\}} \overline{[0,1]} \cup_{\{0\}}[0,1] \cup_{\{1\}} I_{\bullet}\right) \\
& ((s \otimes 1) \circledast(1 \otimes r))(I)=\mathcal{A}\left(\bar{I}_{\circ} \cup_{\{0\}} \overline{[0,1]} \cup_{\{1\}}[0,1] \cup_{\{0\}} \overline{[0,1]} \cup_{\{1\}} \overline{I_{\bullet}}\right),
\end{aligned}
$$

or perhaps more clearly

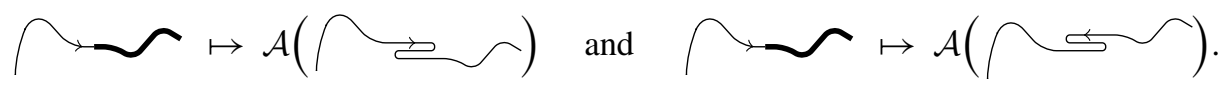

These are isomorphic to the weak units on $\mathcal{A}$ and $\mathcal{A}^{o p}$, and therefore are defects; they are equivalent to identity defects by [BDH19, Remark 1.40 \& Example 3.5].

Assuming $\mathcal{A}$ is finite, we now proceed to show that the unit and counit defects are finite. Let $I_{1}, \ldots, I_{4}$ be as in $(0.1)$; the intervals $I_{1}$ and $I_{3}$ are genuinely bicolored, $I_{2}$ is white, and $I_{4}$ is black. The actions of $r\left(I_{1}\right), r\left(I_{2}\right), r\left(I_{3}\right), r\left(I_{4}\right)$ on the vacuum sector $H_{0}(r)$ are conjugate to the actions of $\mathcal{A}\left(I_{1}\right), \mathcal{A}\left(I_{2}\right) \otimes \mathcal{A}\left(I_{4}\right), \mathcal{A}\left(I_{3}\right)$, and $\mathbb{C}$ on $H_{0}(\mathcal{A})$. The condition of Definition 0.2 then holds by the split property of $\mathcal{A}$.

By the same argument, one also shows that $s$ is a finite defect.

From this theorem and Corollary 2.16, we have the following:

Corollary 2.19. A finite conformal net is fully dualizable.

In any $n$-category, a composition of fully dualizable 1-morphisms is again fully dualizable; similarly a composition (either vertical or horizontal) of fully dualizable 2-morphisms is again fully dualizable. Thus, by Corollary 2.12, the collection of finite sectors is closed under composition, and by Corollary 2.16 and Proposition 2.22 below, the collection of finite defects is closed under composition. By direct inspection, the collection of finite conformal nets is closed under tensor product.

Together, Corollaries 2.12, 2.16, and 2.19 establish the following:

Theorem 2.20. The 3-category of finite conformal nets, finite defects, finite sectors, and intertwiners has all duals.

Applying the cobordism hypothesis (as before under the assumption that it applies to the symmetric monoidal 3-category of conformal nets-see Footnote 3), we obtain the corresponding topological field theories:

Corollary 2.21. Associated to any finite conformal net $\mathcal{A}$, there is a 3-dimensional local framed topological field theory with target the 3-category of conformal nets, whose value on the positively framed point is the conformal net $\mathcal{A}$. 
2.D. Dualizable nets are finite. In the preceding section we saw that the subcategory of finite conformal nets, finite defects, finite sectors, and intertwiners has all duals. In this section, we prove that that this subcategory is in fact the maximal subcategory of the 3-category of conformal nets that has all duals.

We already saw in Corollary 2.12 that a dualizable sector is necessarily finite. We now show that a fully dualizable defect must be finite:

Proposition 2.22. Let $\mathcal{A}$ and $\mathcal{B}$ be finite conformal nets, and let ${ }_{\mathcal{A}} D_{\mathcal{B}}$ be a defect. If $D$ has an adjoint, then $D$ is finite.

Proof. Let $D^{\vee}$ be the dual of $D$, and let $r$ and $s$ be the counit and unit sectors, so that

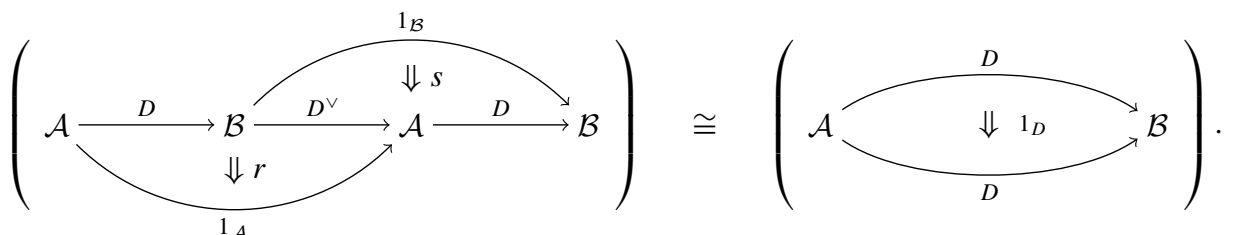

In other words, with $I_{1}, \ldots, I_{13}$ arranged as before

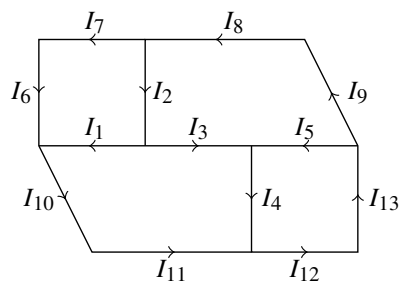

we have

$$
\left(H_{0}(D) \bigotimes_{\mathcal{B}\left(I_{2}\right)} s\right) \underset{D\left(I_{1}\right) \vee D^{\vee}\left(\bar{I}_{3}\right) \vee D\left(I_{5}\right)}{\otimes}\left(r \otimes_{\mathcal{A}\left(I_{4}\right)} H_{0}(D)\right) \cong H_{0}(D) .
$$

We check that $D$ is finite by showing that the action on (2.23) of the algebra $D\left(I_{7}\right) \otimes_{\text {alg }}$ $D\left(I_{12}\right)$ extends to $D\left(I_{7}\right) \bar{\otimes} D\left(I_{12}\right)$.

The Hilbert space $r$ is invertible as a $D\left(I_{1}\right) \vee D^{\vee}\left(I_{3}\right)^{o p} \vee \mathcal{A}\left(I_{4}\right)^{o p}-\left(D\left(I_{1}\right) \vee D^{\vee}\left(I_{3}\right)^{o p} \vee\right.$ $\left.\mathcal{A}\left(I_{4}\right)^{o p}\right)^{\prime}$-bimodule. Similarly, the Hilbert space $s$ is an invertible $\mathcal{B}\left(I_{2}\right) \vee D^{\vee}\left(I_{3}\right) \vee$ $D\left(I_{5}\right)^{o p}-\left(\mathcal{B}\left(I_{2}\right) \vee D^{\vee}\left(I_{3}\right) \vee D\left(I_{5}\right)^{o p}\right)^{\prime}$-bimodule. Fusing (2.23) with the inverse bimodules $\bar{r}$ and $\bar{s}$, and using the (non-canonical) isomorphisms

$$
\begin{aligned}
& L^{2}\left(D\left(I_{1}\right) \vee D^{\vee}\left(I_{3}\right)^{o p} \vee \mathcal{A}\left(I_{4}\right)^{o p}\right) \cong H_{0}\left(D \circledast \mathcal{B} D^{\vee}\right) \\
& L^{2}\left(\mathcal{B}\left(I_{2}\right) \vee D^{\vee}\left(I_{3}\right) \vee D\left(I_{5}\right)^{o p}\right) \cong H_{0}\left(D^{\vee} \circledast \mathcal{A} D\right),
\end{aligned}
$$

we get the Hilbert space

$$
\begin{aligned}
& \left(H_{0}(D) \otimes_{\mathcal{B}} H_{0}\left(D^{\vee} \circledast \mathcal{A} D\right)\right) \underset{D\left(I_{1}\right) \vee D^{\vee}\left(\bar{I}_{3}\right) \vee D\left(I_{5}\right)}{\otimes}\left(H_{0}\left(D \circledast \mathcal{B} D^{\vee}\right) \otimes_{\mathcal{A}} H_{0}(D)\right) \\
& \cong\left(H_{0}(D) \otimes_{\mathcal{B}} H_{0}\left(D^{\vee}\right) \otimes_{\mathcal{A}} H_{0}(D)\right) \underset{D\left(I_{1}\right) \vee D^{\vee}\left(\bar{I}_{3}\right) \vee D\left(I_{5}\right)}{\otimes}\left(H_{0}(D) \otimes_{\mathcal{B}} H_{0}\left(D^{\vee}\right) \otimes_{\mathcal{A}} H_{0}(D)\right) .
\end{aligned}
$$

The latter is isomorphic to

$$
H_{0}(D) \bigotimes_{\mathcal{B}} H_{0}\left(D^{\vee}\right) \bigotimes_{\mathcal{A}} H_{0}(D)
$$


by the interchange isomorphism [BDH19, Section 6.D]. To be precise, letting $J_{1}, J_{2} \ldots, J_{10}$

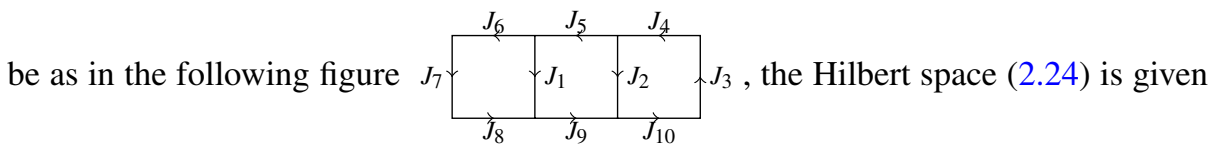
by $H_{0}(D) \bigotimes_{\mathcal{B}\left(J_{1}\right)} H_{0}\left(D^{\vee}\right) \bigotimes_{\mathcal{A}\left(J_{2}\right)} H_{0}(D)$.

The intervals $J_{6}$ and $J_{10}$ correspond to $I_{7}$ and $I_{12}$, respectively. Note that $H_{0}\left(D^{\vee}\right)$ is split as a $\mathcal{B}\left(J_{1}\right)-\mathcal{A}\left(J_{2}\right)$-bimodule. Since the fusion of a split bimodule with any bimodule is always split, it follows that (2.24) is split as a $D\left(J_{6}\right) \vee \mathcal{A}\left(J_{7}\right) \vee D\left(J_{8}\right)-\left(D\left(J_{10}\right) \vee\right.$ $\left.\mathcal{B}\left(J_{3}\right) \vee D\left(J_{4}\right)\right)^{o p}$-bimodule. In particular, it is split as a $D\left(J_{6}\right)-D\left(J_{10}\right)^{o p}$-bimodule. In other words, the completion of $D\left(J_{6}\right) \otimes_{\text {alg }} D\left(J_{10}\right)$ is isomorphic to the spatial tensor product $D\left(J_{6}\right) \bar{\otimes} D\left(J_{10}\right)$.

Finally, we show that fully dualizable conformal nets must be finite. Even though we do not have at hand a 3-category of all (not-necessarily-finite) conformal nets, we do have enough of the structure of that hypothetical 3-category to make sense of the notion of an arbitrary conformal net being fully dualizable, and therefore to make sense of the statement that a fully dualizable not-necessarily-finite conformal net must in fact be finite.

Recall from Theorem 2.17 that any (not-necessarily-finite) conformal net $\mathcal{A}$ has an ambidextrous dual $\mathcal{A}^{o p}$ with evaluation defect $\mathcal{A} \otimes \mathcal{A}^{o p} \mathrm{r}_{\mathbb{C}}$ and coevaluation defect $\mathbb{C}^{\mathrm{S}} \mathcal{A}^{o p} \otimes \mathcal{A}$. We call such a conformal net dualizable if these evaluation and coevaluation defects $r$ and $s$ both have ambidextrous adjoints with dualizable unit and counit sectors. This definition (specifically the notion of an adjunction for the evaluation and coevaluation defects) is well posed because, for any not-necessarily-finite conformal net $\mathcal{B}$ and any defects ${ }_{\mathcal{B}} D_{\mathbb{C}}$ and ${ }_{\mathbb{C}} E_{\mathcal{B}}$, the fusion products $D \circledast_{\mathbb{C}} E$ and $E \circledast_{\mathcal{B}} D$ are indeed defects (the first one by [BDH 19 , Theorem 1.44]; the second one because a $\underline{\mathbb{C}}-\mathbb{C}$-defect is just a von Neumann algebra [BDH19, Proposition 1.22]).

Theorem 2.25. Let $\mathcal{A}$ be a not-necessarily-finite conformal net, and let $r$ and $s$ be the evaluation and coevaluation defects of the duality of $\mathcal{A}$ and $\mathcal{A}^{\text {op }}$, given by $r: I \mapsto$ $\mathcal{A}\left(I_{\circ} \cup_{I_{\bullet}} \bar{I}_{\circ}\right)$ and $s: I \mapsto \mathcal{A}\left(\bar{I}_{\bullet} \cup_{I_{\infty}} I_{\bullet}\right)$. If the defect $r$ has an adjoint $r^{\vee}$, and its counit sector $r \circledast_{\mathbb{C}^{r}} r^{\vee} R_{{\mathcal{A} \otimes \mathcal{A}^{o p}}^{\text {op }}}$ is dualizable, then the conformal net $\mathcal{A}$ is finite.

Note that the proof of this proposition requires particular care: $\mathcal{A}$ is not assumed to have finite index, and so most of our previous results cannot be used here.

Proof. Recall that

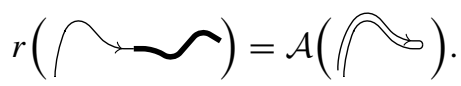

By assumption, $r$ has an adjoint. Let $r^{\vee}$ be its adjoint $\underline{\mathbb{C}}-\left(\mathcal{A} \otimes \mathcal{A}^{o p}\right)$-defect. Let also $r \circledast_{\mathbb{C}} r^{\vee} R_{1_{\mathcal{A} \otimes \mathcal{A}^{o p}}}$ and ${ }_{1_{\mathbb{C}}} S_{r^{\vee} \circledast \mathcal{A}_{\mathcal{A}} \mathcal{A}^{\text {op }} r}$ be the corresponding counit and unit sectors. We now describe the algebras that act on the Hilbert spaces $R$ and $S$.

Take the "standard circle" $\partial[0,1]^{2}$ and cut it open at the point $\left(\frac{1}{2}, 1\right)$. Call the two resulting boundary points $p$ and $q$. The resulting manifold, call it $M$, looks roughly like this:

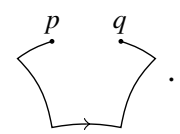


Now consider its doubling $N:=M \cup_{\{p, q\}} \bar{M}$ :

$$
N=\sum^{p},
$$

and let $\kappa: N \rightarrow N$ be the orientation reversing involution that exchanges $M$ and $\bar{M}$ and fixes $p$ and $q$. Given a $\kappa$-invariant neighborhood $J$ of $q$, let $J_{\kappa}:=[0,1] \cup J / \kappa$ be the bicolored interval with bicoloring given by $\left(J_{\kappa}\right)_{\circ}=[0,1]$ and $\left(J_{\kappa}\right)_{\bullet}=J / \kappa$

$$
J=\gg \quad \rightsquigarrow \quad J_{\kappa}=\gg \text {. }
$$

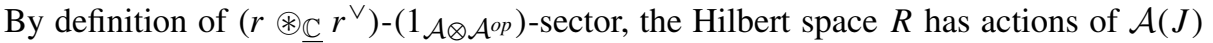
for every subinterval $\bar{J} \subset N$ that avoids $q$, and actions of $r^{\vee}\left(J_{\kappa}\right)$ for every $\kappa$-invariant interval $J$ that contains $q$.

The algebras acting on $S$ are somewhat easier to describe. Consider the double $D:=$ $[0,1] \cup_{\{0,1\}}[0,1]$ of the standard interval $[0,1]$, and let $\kappa: D \rightarrow D$ be the involution that exchanges the two copies of $[0,1]$. The Hilbert space $S$ has an action of $\mathcal{A}(J)$ for every subinterval $J \subset D$ that avoids the point 0 , and an action of $r^{\vee}\left(J_{\kappa}\right)$ for every $\kappa$-invariant interval that contains 0 .

We find it convenient to think of $R$ as being associated to a saddle, and of $S$ as being associated to a cap:
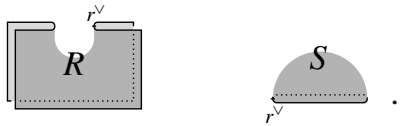

The duality equation

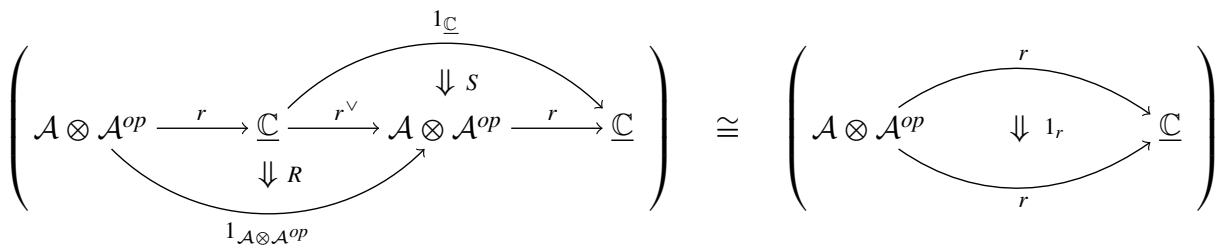

then translates into the statement

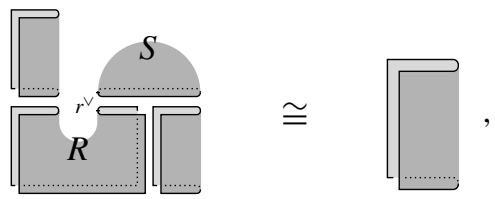

where the left-hand side stands for the fusion of the Hilbert spaces
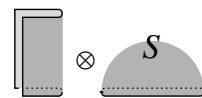
and

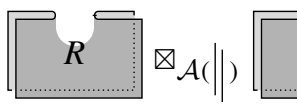
along the algebra

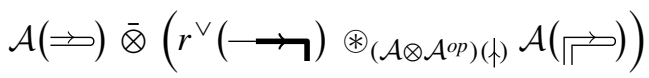




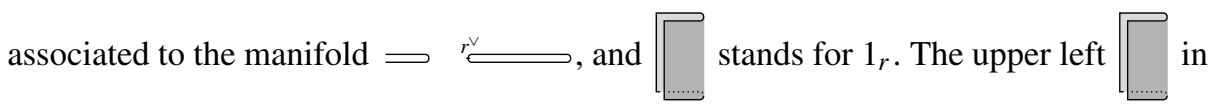
(2.28) does not change anything, and so it can be safely ignored [BDH17, Lemma A.4]. Equation (2.28) then becomes

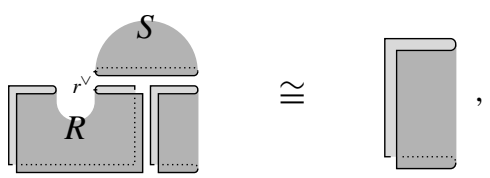

or, equivalently, after flattening the above 2-manifolds:

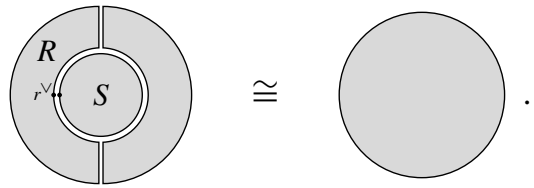

Let us name $I_{1}, \ldots, I_{6}$ the intervals that appear in (2.29)

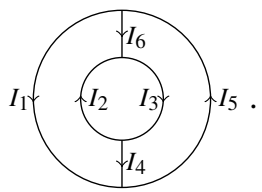

Let $\kappa$ be the reflection in the horizontal axis, and let $K:=\left(I_{2}\right)_{\kappa}=[0,1] \cup I_{2} / \kappa$ be as in (2.27), bicolored by $K_{\circ}=[0,1]$ and $K_{\bullet}=I_{2} / \kappa$. We also abbreviate $H_{0}\left(I_{3} \cup\right.$ $\left.I_{4} \cup I_{5} \cup I_{6}, \mathcal{A}\right)$ by $H_{0}(\mathcal{A})$. The left-hand side of (2.29) stands for the fusion of $S$ with $R \otimes_{\mathcal{A}\left(I_{6} \cup I_{4}\right)} H_{0}(\mathcal{A})$ along the algebra

$$
r^{\vee}(K) \vee \mathcal{A}\left(I_{3}\right)=r^{\vee}\left(K \cup \bar{I}_{6}\right) \circledast\left(\mathcal{A} \otimes \mathcal{A}^{o p}\right)\left(I_{6}\right) \mathcal{A}\left(I_{6} \cup I_{3} \cup I_{4}\right),
$$

where we identify $\left(\mathcal{A} \otimes \mathcal{A}^{o p}\right)\left(I_{6}\right)$ with $\mathcal{A}\left(I_{6} \cup I_{4}\right)$ using the reflection $\kappa: \bar{I}_{6} \cong I_{4}$.

Recall [Lur11, Lecture 21] that a dagger functor $F$ is called 'completely additive' if whenever the collection $\iota_{\alpha}: M_{\alpha} \rightarrow M$ exhibit $M$ as the direct sum $\bigoplus M_{\alpha}$, then also $F\left(\iota_{\alpha}\right): F\left(M_{\alpha}\right) \rightarrow F(M)$ exhibit $F(M)$ as $\bigoplus F\left(M_{\alpha}\right)$. (We called such a functor 'normal' in [BDH19, Appendix B.VIII].) The functor

$$
S_{r^{\vee}(K) \vee \mathcal{A}\left(I_{3}\right)}^{\bigotimes}\left(-\bigotimes_{\mathcal{A}\left(I_{6} \cup I_{4}\right)} H_{0}(\mathcal{A})\right): r^{\vee}\left(K \cup \bar{I}_{6}\right) \text {-modules } \rightarrow \mathcal{A}\left(I_{5}\right) \text {-modules }
$$

is completely additive. It is therefore given by Connes fusion with a certain $r^{\vee}\left(K \cup \bar{I}_{6}\right)^{o p}$ $\mathcal{A}\left(I_{5}\right)$-bimodule [Lur11, Lecture 21]. It then follows from (2.29) that the Hilbert space $R$ is invertible as $r^{\vee}\left(K \cup \bar{I}_{6}\right)-\mathcal{A}\left(I_{1}\right)^{o p}$-bimodule.

Recall that $R$ is finite as $\left(r \circledast \mathbb{C} r^{\vee}\right)-\left(1_{\mathcal{A} \otimes \mathcal{A}^{o p}}\right)$-sector. In other words, it is finite as an

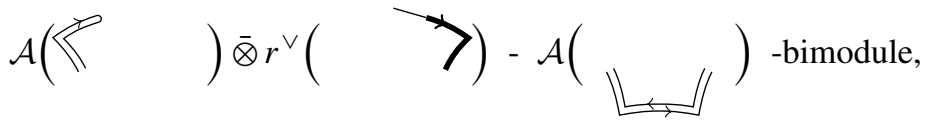


where we again draw our intervals as in (2.26). We know from our previous discussion that $R$ is invertible as an

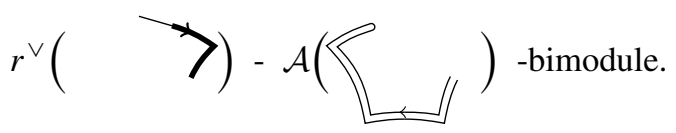

Let $Q$ be the inverse bimodule. Twisting it by a diffeomorphism $\gg \cong$ may treat $Q$ as an

$$
\mathcal{A}(\gg)-r^{\vee}(>) \text {-bimodule. }
$$

By definition, it then satisfies

$$
Q \underset{r^{\vee}(>)}{\otimes} R \cong H_{0}(\longleftarrow, \mathcal{A})
$$

We then also have (applying [BDH17, Lemma A.4])

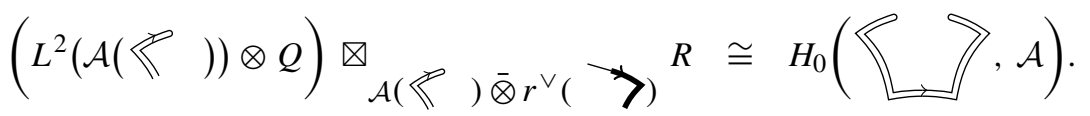

Since $L^{2}(\mathcal{A}(\ll)) \otimes Q$ is an invertible

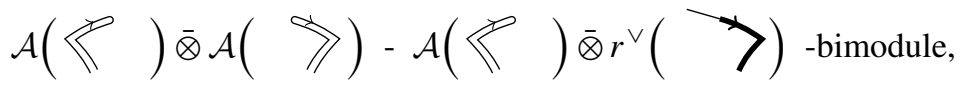

it follows from (2.30) and the finiteness of $R$ that $H_{0}(\square, \mathcal{A})$ is finite as an

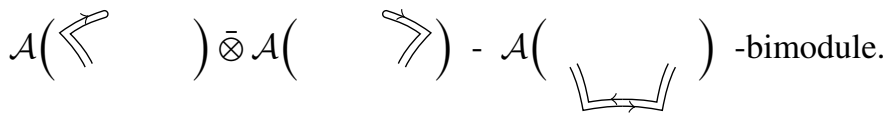

The latter is the definition of what it means for $\mathcal{A}$ to be finite.

Remark 2.31. Recall that strong additivity was assumed as part of our definition of coordinate free conformal nets [BDH15, Definition 1.1].

The above theorem implies that in a hypothetical 3-category of strongly additive notnecessarily-finite-index conformal nets, a fully dualizable conformal net is necessarily finite-index. We expect that even more is true, namely, that in a hypothetical 3-category of not-necessarily-finite-index and not-necessarily-strongly-additive conformal nets, a fully dualizable conformal net is finite-index (and hence strongly additive, by [LX04]). 
Acknowledgements. AB was funded in part by the DFG under Germany's Excellence Strategy EXC 2044390685587. CD was partially supported by a Miller Research Fellowship and by EPSRC grant EP/S018883/1. AH was supported in part by grant VP2-2013-005 from the Leverhulme Trust.

Open Access This article is licensed under a Creative Commons Attribution 4.0 International License, which permits use, sharing, adaptation, distribution and reproduction in any medium or format, as long as you give appropriate credit to the original author(s) and the source, provide a link to the Creative Commons licence, and indicate if changes were made. The images or other third party material in this article are included in the article's Creative Commons licence, unless indicated otherwise in a credit line to the material. If material is not included in the article's Creative Commons licence and your intended use is not permitted by statutory regulation or exceeds the permitted use, you will need to obtain permission directly from the copyright holder. To view a copy of this licence, visit http://creativecommons.org/licenses/by/4.0/.

Publisher's Note Springer Nature remains neutral with regard to jurisdictional claims in published maps and institutional affiliations.

\section{Appendix A: Disintegrating Sectors Between Finite Defects}

Sectors between conformal nets disintegrate into irreducibles [KLM01]; in this section we generalize that result to the case of sectors between defects, provided the defects are finite.

Lemma A.1. Let $\mathcal{A}$ and $\mathcal{B}$ be conformal nets. Let ${ }_{\mathcal{A}} D_{\mathcal{B}}$ and ${ }_{\mathcal{A}} E_{\mathcal{B}}$ be irreducible finite defects. Then any $D$-E-sector disintegrates into a direct integral of irreducible $D$-Esectors.

Proof. Pick a countable collection ${ }^{7}$ of pairs of bicolored subintervals $\left\{I_{i}^{-} \subset I_{i}^{+}\right\}_{i \in \mathcal{I}}$ of the standard bicolored circle, with the closure of $I_{i}^{-}$contained in the interior of $I_{i}^{+}$, satisfying the following conditions:

$-I_{i}^{-}$is genuinely bicolored if and only if $I_{i}^{+}$is genuinely bicolored;

- for all $p, q \in S^{1}$, either

a. there exists an $i \in \mathcal{I}$ such that $p, q \in I_{i}^{-}$, or

b. there exist $i, j \in \mathcal{I}$ such that $p \in I_{i}^{-}, q \in I_{j}^{-}$, and $I_{i}^{+} \cap I_{j}^{+}=\emptyset$.

For each $i \in \mathcal{I}$, let $A_{i}^{ \pm}$denote the algebra $\mathcal{A}\left(I_{i}^{ \pm}\right), \mathcal{B}\left(I_{i}^{ \pm}\right), D\left(I_{i}^{ \pm}\right)$, or $E\left(I_{i}^{ \pm}\right)$depending on whether $I_{i}^{ \pm}$is white, black, contains the top defect point, or contains the bottom defect point, respectively. Because $D$ and $E$ are finite, there exists, for each $i \in \mathcal{I}$, a type $I$ factor $N_{i}$ such that

$$
A_{i}^{-} \subset N_{i} \subset A_{i}^{+}
$$

Let $\mathcal{K}_{i} \subset N_{i}$ denote the ideal of compact operators in $N_{i}$. For each $i, j \in \mathcal{I}$ such that $I_{i}^{+} \cap I_{j}^{+}=\emptyset$, let $R_{i j} \subset \mathcal{K}_{i} * \mathcal{K}_{j}$ be the kernel of the projection $\mathcal{K}_{i} * \mathcal{K}_{j} \rightarrow \mathcal{K}_{i} \otimes \mathcal{K}_{j}$ from the free product $C^{*}$-algebra to the tensor product $C^{*}$-algebra. For each $i, j \in \mathcal{I}$ such that $I_{i}^{+} \subset I_{j}^{-}$, let $S_{i j} \subset \mathcal{K}_{i} * \mathcal{K}_{j}$ be the kernel of the map $\mathcal{K}_{i} * \mathcal{K}_{j} \rightarrow \mathcal{K}_{i} \vee_{N_{j}} \mathcal{K}_{j}$, where $\mathcal{K}_{i} \vee_{N_{j}} \mathcal{K}_{j}$ is the subalgebra of $N_{j}$ generated by $\mathcal{K}_{i}$ and $\mathcal{K}_{j}$. Now define

$$
\left.\mathfrak{A}:=\underset{i}{\left(* \mathcal{K}_{i}\right.}\right) / I
$$

where $I$ is the norm-closed ideal generated by $R_{i j}$ for $i, j \in \mathcal{I}$ such that $I_{i}^{+} \cap I_{j}^{+}=\emptyset$, and $S_{i j}$ for $i, j \in \mathcal{I}$ such that $I_{i}^{+} \subset I_{j}^{-}$.

\footnotetext{
7 In fact this collection can be chosen to be finite.
} 
By Lemma A.2, the category of $D-E$-sectors is equivalent to the category of representations of $\mathfrak{A}$ whose restriction to each $\mathcal{K}_{i}$ is nondegenerate.

Because $\mathfrak{A}$ is a separable $C^{*}$-algebra, the category $\operatorname{Rep}(\mathfrak{A})$ admits direct integral decompositions. We need to show that given a representation $H$ of $\mathfrak{A}$ whose restriction to each $\mathcal{K}_{i}$ is nondegenerate, and a direct integral decomposition $(H, \rho) \cong \int_{x \in X}\left(H^{x}, \rho^{x}\right) d x$, almost all of the integrands $\left(H^{x}, \rho^{x}\right)$ again have the property that their restriction to each $\mathcal{K}_{i}$ is nondegenerate. Pick an increasing sequence of projections $p_{n}^{i} \in \mathcal{K}_{i}$, $n \in \mathbb{N}$, that forms an approximate unit. By Lemma A.3, we have that $1=\sup \rho_{i}\left(p_{n}^{i}\right)=$ $\sup \int^{\oplus} \rho_{i}^{x}\left(p_{n}^{i}\right)=\int^{\oplus} \sup \rho_{i}^{x}\left(p_{n}^{i}\right)$. This implies that for almost all $x$, we have $\sup \rho_{i}^{x}\left(p_{n}^{i}\right)=$ 1.

Lemma A.2. The category of representation of $\mathfrak{A}$ whose restriction to each $\mathcal{K}_{i}$ is nondegenerate is equivalent to the category of D-E-sectors.

Proof. By construction, every $D-E$-sector yields an appropriate representation of $\mathfrak{A}$. Now suppose that we have a representation of $\mathfrak{A}$ on a Hilbert space $H$ whose restriction to each $\mathcal{K}_{i}$ is nondegenerate. By the classification of the representations of compact operators, the action of $\mathcal{K}_{i}$ extends uniquely to a normal action $\rho_{i}: N_{i} \rightarrow B(H)$. For every $i, j \in \mathcal{I}$ such that $I_{i}^{+} \cap I_{j}^{+}=\emptyset$, the action of $\mathcal{K}_{i} * \mathcal{K}_{j}$ descends to an action of $\mathcal{K}_{i} \otimes \mathcal{K}_{j}$; by the ultraweak density of $\mathcal{K}_{i}$ in $N_{i}$, the actions of $N_{i}$ and $N_{j}$ commute. Now, for every $i, j \in \mathcal{I}$ such that $I_{i}^{+} \subset I_{j}^{-}$, the action of $\mathcal{K}_{i} * \mathcal{K}_{j}$ descends to an action of $\mathcal{K}_{i} \vee_{N_{j}} \mathcal{K}_{j}$. By [KLM01, Corollary 53], that action of $\mathcal{K}_{i} \vee_{N_{j}} \mathcal{K}_{j}$ extends uniquely to a normal action $\tilde{\rho}_{j}: N_{j} \rightarrow B(H)$, which agrees with $\rho_{j}$ by the ultraweak density of $\mathcal{K}_{j}$ inside $N_{j}$. We therefore have a diagram

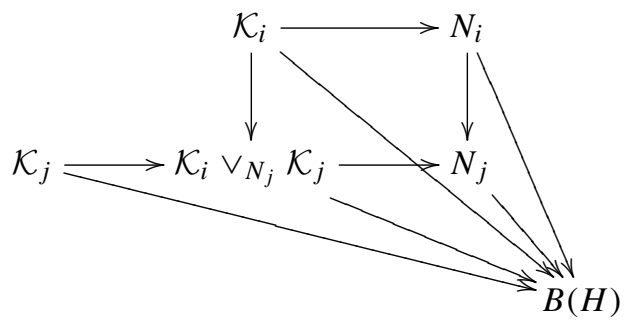

where all triangles are known to commute except possibly the triangle with edge $N_{i} \rightarrow$ $N_{j}$. The missing triangle commutes because $\mathcal{K}_{i}$ is ultraweakly dense in $N_{i}$. Therefore, by [BDH19, Lemma 2.5], the actions $\left.\rho_{i}\right|_{A_{i}^{-}}$assemble into a $D-E$-sector structure on $H$.

Lemma A.3. Let $H_{x}$ be a measurable family of Hilbert spaces over a probability space $X$. For each $n \in \mathbb{N}$, let $p_{n, x} \in B\left(H_{x}\right)$ be a measurable family of projections indexed by the points of $X$. Assume furthermore that for every $x \in X$, the sequence $\left\{p_{n, x}\right\}_{n \in \mathbb{N}}$ is increasing. Then

$$
\int^{\oplus} \sup p_{n, x}=\sup \int^{\oplus} p_{n, x} .
$$

Proof. Let $M \subset B(H)$ be the abelian von Neumann algebra on $H:=\int^{\oplus} H_{x}$ generated by $\int^{\oplus} f(x) p_{n, x}$ for all $f \in L^{\infty}(X)$ and $n \in \mathbb{N}$. Note that $M \cong L^{\infty}(Y)$ for some measure space $Y$. Since $L^{\infty}(X) \subset M$, we have a measurable map $\pi: Y \rightarrow X$ and we can write $M=\int_{X}^{\oplus} M_{x}$, where $M_{x}=L^{\infty}\left(\pi^{-1}(x)\right)$. The projections $p_{n, x} \in M_{x}$ correspond 
to measurable subsets $Z_{n, x} \in \pi^{-1}(x)$, and the equation $\int^{\oplus} \sup p_{n, x}=\sup \int^{\oplus} p_{n, x}$ follows from the fact that $\bigsqcup_{x} \bigcup_{n} Z_{n, x}=\bigcup_{n} \bigsqcup_{x} Z_{n, x}$.

\section{Appendix B: A Variant Vertical Composition}

In [BDH19, §2.C], we defined the vertical composition of two sectors ${ }_{D} H_{E}$ and ${ }_{E} K_{F}$ to be the fusion along half of each 'circle', $H \bigotimes_{E\left(S_{+}^{1}\right)} K$, with the evident remaining actions of $D$ and $F$ :

$$
{ }_{D} H \otimes_{E} K_{F}=\text { fusion }_{\mathrm{v}}(\mathcal{A} \underset{E}{H} \mathcal{B}, \mathcal{A}
$$

An alternative definition would be to fuse along a 'quarter-circle':

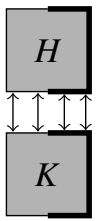

and to equip the resulting Hilbert space with the structure of a $D-F$-sector by means of a diffeomorphism

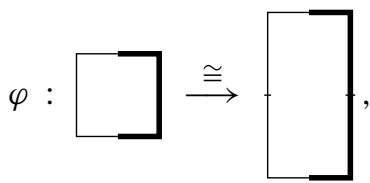

compatible with the local coordinates around the color-change points. Specifically, the resulting sector is $\varphi^{*}\left(H \bigotimes_{E(I)} K\right)$, where $I$ is the top quarter of the circle (associated to the sector $K$ ), or equivalently the bottom quarter of the circle (associated to the sector $H)$.

Lemma B.3. Let ${ }_{D} H_{E}$ and ${ }_{E} K_{F}$ be sectors, and let $\varphi$ be a diffeomorphism from the standard circle to the larger circle, as above. Then the vertical fusion $H \bigotimes_{E\left(S_{\top}^{1}\right)} K$ from (B.1) is (non-canonically) isomorphic, as a D-F-sector, to the alternative fusion $\varphi^{*}\left(H \bigotimes_{E(I)} K\right)$ from (B.2).

Proof. Let $\psi_{1}: S^{1} \rightarrow S^{1}$ be a diffeomorphism which maps the lower semi-circle $S_{\perp}^{1}$ to the lower quarter-circle (drawn here as an edge of a square) and satisfies $\left.\varphi\right|_{S_{\top}^{1}}=\left.\psi_{1}\right|_{S_{\top}^{1}}$, let $\psi_{2}: S^{1} \rightarrow S^{1}$ be a diffeomorphism which maps the upper semi-circle $S_{\perp}^{1}$ to the upper quarter-circle and satisfies $\left.\varphi\right|_{S_{\perp}^{1}}=\left.\psi_{2}\right|_{S_{\perp}^{1}}$, and let $u_{\psi_{1}}$ and $u_{\psi_{2}}$ be unitaries implementing these diffeomorphisms (these exist by [BDH19, Proposition 1.10]). We assume without loss of generality that $\psi_{2}=j \circ \psi_{1} \circ j$, where $j$ is the reflection along the horizontal axis of symmetry. Then $u_{\psi_{1}} \otimes u_{\psi_{2}}$ maps $H \bigotimes_{E\left(S_{\top}^{1}\right)} K$ to $H \bigotimes_{E(I)} K$, and is an isomorphism of $D$ - $F$-sectors $H \bigotimes_{E\left(S_{\top}^{1}\right)} K \cong \varphi^{*}\left(H \bigotimes_{E(I)} K\right)$. 


\section{References}

[AF17] Ayala, D., Francis, J.: The cobordism hypothesis. arXiv:1705.02240 (2017)

[BD95] Baez, J.C., Dolan, J.: Higher-dimensional algebra and topological quantum field theory. J. Math. Phys. 36(11), 6073-6105 (1995)

[BDH14] Bartels, A., Douglas, C.L., Henriques, A.: Dualizability and index of subfactors. Quantum Topol. 5, 289-345. arXiv:1110.5671 (2014)

[BDH15] Bartels, A., Douglas, C.L., Henriques, A.: Conformal nets I: coordinate-free nets. Int. Math. Res. Not. 13, 4975-5052. arXiv:1302.2604 (2015)

[BDH17] Bartels, A., Douglas, C.L., Henriques, A.: Conformal nets II: conformal blocks. Commun. Math. Phys. 354, 393-458. arXiv:1409.8672 (2017)

[BDH18] Bartels, A., Douglas, C.L., Henriques, A.: Conformal nets IV: the 3-category. Algebr. Geom. Top. 18, 897-956. arXiv:1605.00662 (2018)

[BDH19] Bartels, A., Douglas, C.L., Henriques, A.: Fusion of defects (formerly "Conformal nets III: fusion of defects”). Mem. Am. Math. Soc. 1237, 1-108. arXiv:1310.8263 (2019)

[BK01] Bakalov, B., Kirillov, A., Jr.: Lectures on Tensor Categories and Modular Functors, University Lecture Series, vol. 21. American Mathematical Society, Providence (2001)

[CS15] Calaque, D., Scheimbauer, C.: A note on the $(\infty, n)$-category of cobordisms. arXiv:1509.08906 (2015)

[DSPS17] Douglas, C.L., Schommer-Pries, C., Snyder, N.: Dualizable tensor categories. Mem. Am. Math. Soc. arXiv:1312.7188 (2017)

[Gui18] Gui, B.: Categorical extensions of conformal nets. arXiv:1812.04470 (2018)

[Hen17] Henriques, A.: What Chern-Simons theory assigns to a point. Proc. Natl. Acad. Sci. U. S. A. 114(51), 13418-13423 (2017)

[KLM01] Kawahigashi, Y., Longo, R., Müger, M.: Multi-interval subfactors and modularity of representations in conformal field theory. Commun. Math. Phys. 219(3), 631-669 (2001)

[Lur09] Lurie, J.: On the classification of topological field theories. In: Current Developments in Mathematics, pp. 129-280. International Press, Somerville (2008)

[Lur11] Lurie, J.: Lecture notes on von Neumann algebras. https://www.math.harvard.edu/ lurie/261y. html (2011)

[LX04] Longo, R., Xu, F.: Topological sectors and a dichotomy in conformal field theory. Commun. Math. Phys. 251(2), 321-364 (2004)

Communicated by Y. Kawahigashi 